\title{
TRIM71 deficiency causes germ cell loss during mouse embryogenesis and promotes human male infertility
}

Lucia A. Torres-Fernández ${ }^{1 \dagger}$, Jana Emich ${ }^{2 \dagger}$, Yasmine Port ${ }^{1 \dagger}$, Sibylle Mitschka ${ }^{1}$, Marius Wöste $^{3}$, Simon Schneider ${ }^{4}$, Daniela Fietz ${ }^{5}$, Manon S. Oud ${ }^{6}$, Sara Di Persio ${ }^{7}$, Nina Neuhaus ${ }^{7}$, Sabine Kliesch ${ }^{8}$, Michael Hölzel ${ }^{9}$, Hubert Schorle ${ }^{4}$, Corinna Friedrich ${ }^{2}$, Frank Tüttelmann ${ }^{2+^{*}}$ and Waldemar Kolanus ${ }^{1 \dagger^{*}}$

${ }^{1}$ Life and Medical Sciences Institute, University of Bonn, 53115 Bonn, Germany

${ }^{2}$ Institute of Reproductive Genetics, University of Münster, 48149 Münster, Germany

3Institute of Medical Informatics, University of Münster, 48149 Münster, Germany

${ }^{4}$ Institute of Pathology, University Hospital Bonn, 53127 Bonn, Germany

5 Institute for Veterinary Anatomy, Histology and Embryology, and Hessian Centre of Reproductive Medicine (HZRM). Justus Liebig University Gießen, 35392 Gießen, Germany.

${ }^{6}$ Department of Human Genetics, Radboud university medical center, Nijmegen, The Netherlands

${ }^{7}$ Centre of Reproductive Medicine and Andrology, Institute of Reproductive and Regenerative Biology, University Hospital Münster, 48149 Münster, Germany

${ }^{8}$ Centre of Reproductive Medicine and Andrology, Department of Clinical and Surgical Andrology, University Hospital Münster, 48149 Münster, Germany

9Institute for Experimental Oncology, University Hospital Bonn, 53127 Bonn, Germany

†Equal contribution

*Correspondence: wkolanus@uni-bonn.de and Frank.Tuettelmann@ukmuenster.de

\begin{abstract}
Mutations affecting the germline can result in infertility or the generation of germ cell tumors (GCT), highlighting the need to identify and characterize the genes controlling the complex molecular network orchestrating germ cell development. TRIM71 is a stem cell-specific factor essential for embryogenesis, and its expression has been reported in GCT and adult mouse testes. To investigate the role of TRIM71 in mammalian germ cell embryonic development, we generated a germline-specific conditional Trim71 knockout mouse (CKO) using the early primordial germ cell (PGC) marker Nanos3 as a Cre-recombinase driver. cKO mice are infertile, with male mice displaying a Sertoli cell-only (SCO) phenotype, which in humans is defined as a specific subtype of non-obstructive azoospermia characterized by the absence of developing germ cells in the testes' seminiferous tubules. Infertility originates during embryogenesis, as the SCO phenotype was already apparent in neonatal mice. The in vitro differentiation of mouse embryonic stem cells (ESCs) into PGC-like cells (PGCLCs) revealed reduced numbers of PGCLCs in Trim71deficient cells. Furthermore, in vitro growth competition assays with wild type and CRISPR/Cas9generated TRIM71 mutant NCCIT cells, a human GCT-derived cell line which we used as a surrogate model for proliferating PGCs, showed that TRIM71 promotes NCCIT cell proliferation and survival. Our data collectively suggest that germ cell loss in cKO mice results from combined defects during the specification and maintenance of PGCs prior to their sex determination in the genital ridges. Last, via exome sequencing analysis, we identified several TRIM71 variants in a cohort of infertile men, including a loss-of-function variant in a patient with SCO phenotype. Our work reveals for the first time an association of TRIM71 variants with human male infertility, and uncovers further developmental roles for TRIM71 in the generation and maintenance of germ cells during mouse embryogenesis.
\end{abstract}




\section{Introduction}

Despite the recent insights into the regulation of mammalian germ cell development and the advances in assisted reproductive technologies, infertility remains a common problem in modern society affecting $\sim 15 \%$ of couples in industrialized countries ${ }^{1}$. Genetic defects during germ cell development can drive infertility and increase susceptibility to germ cell tumors $(\mathrm{GCT})^{2-4}$.

Primordial germ cells (PGCs) are the first established germ cell population during embryonic development. In mice, PGC specification is initiated in the post-implantation epiblast at embryonic day (E) 6.25 in response to BMP4 signaling from the extra-embryonic ectoderm ${ }^{5}$. BMP4 activates the expression of Prdm14 ${ }^{6}$ and Prdm1/Blimp1 ${ }^{7}$. BLIMP1 then activates the expression of Tfap2 ${ }^{8}$, and together BLIMP1, TFAP2C and PRDM14 establish the transcriptional program required for PGC specification ${ }^{9}$ which is completed at E7.5. At this time point, about 40 cells in the proximal epiblast express early PGC-specific markers such as Nanos $3^{10,11}$. PGCs then migrate to the genital ridges (developing gonads) while slowly proliferating ${ }^{12,13}$. At E10.5, around 1000 PGCs colonize the genital ridges, upregulate late PGC markers such as $D d x 4 / \operatorname{Vasa}^{14,15}$ and $G c n a 1^{16}$, and undergo significant proliferation before initiating sex determination ${ }^{17,18}$. Importantly, PGCs that fail to reach the genital ridges or to further differentiate into gametes are the origin of GCT, which often affect children and young adults ${ }^{2-4,19}$. Therefore, unravelling the processes controlling germline development will not only contribute to our understanding of infertility, but may also offer unique opportunities for the treatment of GCT.

Tripartite Motif Containing 71 (TRIM71) belongs to the TRIM-NHL family and is a stem cell-specific protein ${ }^{20}$ which can act both as an E3 ubiquitin ligase ${ }^{21-24}$ and an mRNA repressor ${ }^{25-29}$. TRIM71 function is essential for embryogenesis and its expression is mostly restricted to early proliferative developmental stages, being downregulated in the course of differentiation ${ }^{30-33}$. However, a postnatal Trim71 expression has also been observed in adult mouse testes ${ }^{33,34}$ as well as in several GCT-derived cell lines ${ }^{25,29,33}$, and a recent study has reported a postnatal function for TRIM71 in adult mouse spermatogenesis ${ }^{34}$. Furthermore, RNA sequencing of Trim71-deficient mouse embryonic stem cells (ESCs) revealed a decreased expression of genes associated with reproductive processes ${ }^{28}$, suggesting an early developmental role for TRIM71 in the germline.

To elucidate the role of TRIM71 in fertility and mammalian embryonic germ cell development, we generated a mouse model with an early germline-specific depletion of Trim 71 driven by the Nanos3-Cre promoter. Additionally, we employed an in vitro approach for the differentiation of wild type and Trim71deficient ESCs into PGCs in order to study the role of TRIM71 during PGC specification. Furthermore, we generated TRIM71 mutant NCCIT cells via CRISPAR/Cas9 to investigate the role of TRIM71 in the proliferation of GCT-derived cells. Last, we used exome sequencing data from infertile men and developed a novel software tool named Haystack to search for novel genetic causes of infertility. Our work thereby identifies TRIM71 as a novel gene associated with human male infertility, and uncovers further developmental roles for TRIM71 in the generation and maintenance of germ cells during mouse embryogenesis. 


\section{Results}

\section{Trim71 is expressed in spermatogonial stem cells and is essential for mouse fertility}

Mice carrying a Trim71 homozygous deletion (KO, Trim $71^{-1}$ ) die during embryonic development (Suppl. Fig. $1 \mathrm{~A})^{31,32}$. In contrast, Trim 71 heterozygous mice (HET, Trim $71^{f /-}$ ) are viable and fertile, although significantly smaller in length and weight than wild type (WT, Trim $\left.71^{f / f f}\right)$ littermates (Suppl. Fig. 1B-C). We detected Trim71 expression in the testes of wild type and heterozygous adult mice - but not in other organs such as heart or kidney - with Trim 71 mRNA levels decreased in the testis of heterozygous mice (Fig. 1A). We then measured the weight of several organs relative to the total body weight, and observed that testes - but neither heart nor kidney - were significantly smaller in heterozygous males compared to wild type males (Fig. 1B). Accordingly, sperm counts were significantly reduced in adult heterozygous males (Fig. 1C). These data suggest that Trim71 expression levels are important for male gonad development and reveal a haploinsufficiency of Trim71 in mice.

Since TRIM71 is a stem cell-specific protein, we hypothesized that its expression in mice testes is present in undifferentiated spermatogonia, also known as spermatogonial stem cells (SSCS). To validate this hypothesis, we used wild type testes cell suspensions to enrich SSCs (THY1.2+) via magnetic-activated cell sorting (MACS) ${ }^{35}$. We found an increase of Trim 71 mRNA as well as the mRNAs of the known SSC-specific markers Sall $4^{36}$ and $\operatorname{Lin} 28 a^{37}$ - but not the differentiating spermatogonia marker Lin28 ${ }^{38}$ - in the THY1.2+ enriched cell population (Fig. 1D-E). This result indicates that Trim71 expression in adult mouse testes is enriched in SSCs. Indeed, single-cell RNA sequencing of mouse adult testicular tissue confirms a restricted expression of Trim71 in SSCs (Fig. 1F-H) ${ }^{39}$.

To study the function of Trim71 in male gonad development, a germline-specific Trim71 conditional knockout mouse (CKO) was generated with the Cre recombinase expressed under the promoter of the early PGC marker Nanos3 (Suppl. Fig. 2A). For a first functional evaluation, adult cKO mice (Trim71 ${ }^{\text {fl/-; }}$ Nanos $3^{\mathrm{Cre} /+}$ ) were crossed with wild type mice. Trim 71 deficiency in the germline resulted in infertility in both sexes, as neither males nor females were able to produce offspring (Suppl. Fig. 2B).

\section{Germline-specific Trim71 cKO male mice display a Sertoli cell-only (SCO)-like phenotype}

Macroscopic analysis of cKO male and female reproductive organs revealed a dramatic reduction in testis and ovary size, respectively (Fig. 2A-C and Suppl. Fig. 2C). Hematoxylin and eosin (H\&E) staining of adult male testis cross-sections showed that most seminiferous tubules in cKO testis were lacking signs of spermatogenesis (Fig. 2D). Furthermore, immunofluorescence staining of germ cells (GCNA1 ${ }^{40,41}$ ) and Sertoli cells (WT1 ${ }^{42}$ ) showed a dramatic reduction of seminiferous tubules containing GCNA+ cells in cKO mice (Fig. 2E-F). Accordingly, the expression of SSC-specific markers Oct4, Sall4 and Lin28a, as well as the differentiating spermatogonia marker Lin28b, were dramatically reduced in whole testis RNA of cKO mice (Fig. 2G), representing the deficit of germ cells in these mice.

This phenotype is reminiscent of a human condition known as Sertoli cell-only (SCO) phenotype, which is a specific type of non-obstructive azoospermia characterized by a total or substantial absence of germ cells in the seminiferous tubules, and whose causes remain mostly unknown ${ }^{43-45}$. Our results here suggest that Trim71 cKO male mice display an SCO-like phenotype with most seminiferous tubules lacking germ cells. 


\section{Infertility in germline-specific Trim71 cKO male mice has an embryonic origin}

As Nanos3 expression in mice embryos is detected as early as E7.5 during PGC specification ${ }^{11}$, and sex determination of PGCs does not occur until E10.5 ${ }^{17,18}$, the infertility observed in both male and female Trim71 cKO mice may reflect a function of TRIM71 in an early stage of germ cell development. In order to determine whether the deficit of germ cells derives from defects during embryonic development, we conducted H\&E staining and GCNA1-WT1 immunostaining on testis cross-sections from neonatal (P0.5) male mice (Fig. 3A-B). Indeed, the SCO-like phenotype previously observed in our adult cKO male mice was already apparent in neonatal cKO mice with a reduction in gonocyte-containing seminiferous tubules (Fig. 3B-C). A recent study has shown that postnatal Trim71 expression in male mouse is important for the successful establishment and differentiation of the adult SSC pool ${ }^{34}$. Our results here additionally show that Trim 71 expression during embryonic development is required for the generation and/or maintenance of the PGC/gonocyte pool before birth.

\section{TRIM71 is required for the optimal differentiation of ESCs into PGCs in vitro}

In order to determine whether Trim71 deficiency causes PGC specification defects, wild type (WT, Trim $\left.71^{f / f f}\right)$ and Trim71 knockout (KO, Trim $71^{\%}$ ) murine $\mathrm{ESCs}^{28}$ were differentiated into PGC-like cells (PGCLCs) in vitro as previously described ${ }^{46}$. In short, ESCs (d0) growing under naïve conditions were primed to EpiSCs (d2) for two days in monolayer culture and differentiated into PGCLCs (d8) for six more days in the presence of BMP4 while growing as spheroids (Fig. 4A). ESCs (d0) represent in vivo embryonic stem cells of the blastocyst's inner cell mass at E3.5-4.5 while EpiSCs (d2) represent in vivo epiblast stem cells at E5.5-6.5, and PGCLCs (d8) should correspond to in vivo early PGCs at E7.5 ${ }^{46}$. Thus, several markers were measured in bulk cell populations at d0, $d 2$ and $d 8$ to monitor the differentiation process (Suppl. Fig. 3).

Both wild type and Trim71-deficient ESCs were primed to EpiSCs as shown by a decrease of the naïve pluripotency marker $K I f 4^{46,47}$ and a simultaneous increase of the primed pluripotency epiblast-specific marker Dnmt3 $b^{46,48}$ at d2 (Suppl. Fig. 3A-B). The subsequent Dnmt3b downregulation observed at d8 was indicative of a successful specification induction by BMP4, as the early PGC marker Prdm14 represses Dnmt3b to enable PGCs epigenetic reprogramming ${ }^{49,50}$. Accordingly, both wild type and Trim71-deficient cells showed a significant upregulation of PGC-specific markers at d8 compared to d2 including Prdm14, Prdm1/Blimp1 and their downstream targets Tfap2c and Nanos3 (Suppl. Fig. 3C-F). However, the levels of Prdm1/Blimp1 at d8 were significantly lower in Trim71-deficient cells than in wild type cells, an effect that was also apparent - although not significant - for Nanos3, suggesting a moderate impairment in the specification of Trim71-deficient PGCLCs. Importantly, while an upregulation of the early PGC marker Nanos $3^{11}$ was already observable at $\mathrm{d} 8$, the expression of the late PGC marker Ddx4/Vasa ${ }^{14,15}$ was not increased (Suppl. Fig. 3G), indicating that the in vitro-generated PGCLCs represent in vivo pre-migratory PGCs at the end of the specification phase (E7.5), as expected.

In order to estimate the number of PGCLCs specified from wild type and Trim71-deficient ESCs, d8-bulk populations were stained for the surface markers ITGB3/CD61 (PE-Cy7) and SSEA-1/CD15 (APC) previously reported to unequivocally identify PGCLCs ${ }^{46}$ - and were analyzed by flow cytometry. We found that the number of PGCLCs derived from Trim71-deficient ESCs was reduced by about $25 \%$ (Fig. 4B-C). A significant decrease of the PGC-specific marker SSEA-1/CD15 (APC) - but not of ITGB3/CD61 (PE-Cy7) was also observed in PGCLCs derived from Trim71-deficient ESCs (Fig. 4D-E). 
Of note, Oct4 is required for the maintenance of PGCs after their specification ${ }^{51}$. The highest expression of Oct4 in the mouse embryo is observed in the early blastocyst and decreases progressively in the epiblast upon gastrulation until being restricted to PGCs at E7.5 20,52. Accordingly, we detected a high Oct4 expression in ESCs (d0) which significantly decreased upon priming to EpiSCs (d2) and was maintained in PGCLCs (d8) upon specification (Suppl. Fig. 3H). A similar pattern was observed for Trim71 expression in wild type cells (Suppl. Fig. 3I) with a decrease in its mRNA level after priming (d2) and a sustained expression in PGCLCs (d8). While our results have revealed a moderate impairment in the specification of Trim71-deficient PGCLCs, we believe that this effect may not fully explain the dramatic phenotype observed in Trim71 cKO mice. This, together with the sustained Trim71 expression in PGCLCs at d8 suggest a further role for Trim71 downstream of PGC specification.

\section{TRIM71 controls proliferation in the germline-derived tumor cell line NCCIT}

After specification (E7.5), PGCs migrate and colonize the genital ridges. PGCs then actively proliferate before undergoing sex determination (E10.5), followed by mitotic arrest (E13.5) in case of male PGCs now termed gonocytes. Thus, the cluster of $\sim 40$ founder PGCs grows to approximately 25,000 gonocytes in the developing male gonads during this period ${ }^{12,13,17,18}$. Importantly, the failure of male PGCs to further differentiate into gametes can lead to the development of testicular GCT (TGCT) ${ }^{2-4}$.

TRIM71 is highly expressed in several GCT-derived cell lines ${ }^{25,29,33}$ (Suppl. Fig. 4A) and found to be upregulated in TGCT patients ${ }^{53}$ (Supp. Fig. 4B). We therefore used the GCT-derived embryonic carcinoma cell line NCCIT to generate TRIM71 frameshift mutations via CRISPR/Cas9 and evaluated whether TRIM71 controls NCCIT proliferation in growth competition assays. TRIM71 mutations were generated by using two different single guide RNAs (sgRNA), one targeting the N-terminal RING domain and another targeting the C-terminal NHL domain (Suppl. Fig. 4C). For the RING sgRNA ( $\triangle$ RING), the generation of single NCCIT clones showed that an $83 \mathrm{kDa}$ N-truncated RINGless version of TRIM71 is generated from the use of an alternative in-frame ATG codon present downstream of the targeting region (Suppl. Fig. 4D). For the NHL sgRNA ( $\triangle N \mathrm{NH} 6$ ), generation of single clones showed that the resultant protein was either absent or had a C-terminal truncation of the last NHL repeat (Suppl. Fig. $4 \mathrm{E}$ ), a mutation which is already known to mimic the full KO phenotype in vivo ${ }^{31}$ and in vitro ${ }^{29}$.

Single cell clones often have a selection bias towards robust in vitro proliferation and survival. In order to study TRIM71-dependent cell proliferation in an unbiased manner, we analyzed cell population dynamics during in vitro growth competition assays using non-clonal mixed pools of wild type and TRIM71 mutant cells. To this end, NCCIT cells were mock-transfected with an empty vector (EV) or with TRIM71-targeting vectors ( $\triangle \mathrm{RING}$ or $\triangle \mathrm{NHL6}$ ) and sorted for Cas9-GFP+ cells. Bulk-transfected EV (wild type) and $\triangle$ RING/DNHL6 (TRIM71 mutant) NCCIT pure populations (Suppl. Fig. 5A-B) were mixed in a 1:1 ratio. We then evaluated changes in the distribution of single reads via NGS (Illumina MiSeq system) ${ }^{54}$ for wild type and TRIM71 mutant alleles over a time period of 21 days and classified them as wild type reads, reads with TRIM71 frameshift (loss-of-function) mutations and reads with TRIM71 in-frame mutations (Fig. 5). As a negative control, pure wild type NCCIT (EV) cell populations were analyzed at day 0 and day 21, showing no relevant changes in allele distribution (Suppl. Fig. 5C-D). In contrast, the percentages of alleles with TRIM71 frameshift mutations dropped 2-fold (from $11.34 \%$ to $5.96 \%$ ) for NCCIT $\triangle$ RING cells within 21 days (Fig. 5A) and $\sim 13$-fold (from $32.46 \%$ to $2.53 \%$ ) for NCCIT $\Delta$ NHL6 cells in the same time period (Fig. 5B). Interestingly, TRIM71 in-frame mutations also resulted in a growth disadvantage, although to a lesser degree in each case (Fig. 5A-B). 
These results demonstrate that TRIM71 is involved in the control of proliferation in NCCIT cells, consistent with previous studies involving other tumor cell lines ${ }^{22,24,29,55}$. Our work, together with the elevated TRIM71 expression observed in TGCT patients, confirms a role for TRIM71 supporting the proliferation/maintenance of TGCT. Considering that PGCs undergo active proliferation after reaching the genital ridges, and that TGCT derive from PGCs which fail to further develop into gonocytes, the deficit of germ cells observed in our Trim 71 cKO male mouse possibly results from a combination of specification defects and subsequent proliferation defects in PGCs upon TRIM71 ablation.

\section{Exome sequencing data identifies TRIM71 variants in infertile men with severely impaired spermatogenesis}

The causes of the SCO phenotype in men remain poorly understood ${ }^{43-45}$. In order to identify novel associated genes, we utilized exome sequencing data of 247 SCO subjects belonging to the Male Reproductive Genomics (MERGE) study. We employed our in-house software Sciobase and our newly developed software Haystack to analyze loss-of-function (LoF) variants. After strict filtering based on quality criteria, minor allele frequency (MAF) in the general population, high expression in human testes (GTEx) and absence of LoF variants in individuals with complete spermatogenesis, we identified a total of 721 genes with LoF variants specifically found in SCO patients (Fig. 6A-C). Via this independent and unbiased approach, we found TRIM71 to be present among these genes, revealing a possible association with human male infertility. This finding is supported by our previous data showing that TRIM71 deficiency results in an SCO-like phenotype in male mice. Of note, most of the identified human genes carried LoF variants in only one patient within the studied cohort and none of the identified genes carried LoF variants in more than four individuals, indicating that the SCO phenotype is highly heterogeneous (Fig. 6B). Similar results were observed upon inclusion of genes with lower expression in the testis (Fig. 6C).

For a further evaluation of TRIM71 as a candidate gene associated with human male infertility, we screened 908 infertile patients - including those with SCO from MERGE - for variants in TRIM71. We identified 11 different rare (MAF $\leq 0.001$ ) heterozygous TRIM71 variants, including the aforementioned LoF variant and 10 novel missense variants, present in a total of 14 infertile individuals with severely impaired spermatogenesis and varying histological phenotypes (Fig. 6D-F and Table 1). The majority of subjects (11 out of 14) were azoospermic, and some of them presented with reduced testicular volumes, elevated luteinizing hormone (LH) levels, elevated follicle-stimulating hormone (FSH) levels and/or reduced serum testosterone $(T)$ levels (Table 1 ), all signs of broad testicular dysfunction present in $60 \%$ of azoospermic men ${ }^{44}$.

Generally, TRIM71 seems to be rather intolerant to missense variation (Figure 6D) ${ }^{56}$. This was also indicated by a high $Z$ score value of 3.28 for TRIM71. The $Z$ score is a metric computed by gnomAD database and ranges from -5 to 5 , with higher values indicating an intolerance to variation and, therefore, a higher likelihood for TRIM71 variants to disrupt TRIM71 function. Furthermore, parameters such as MAF or pathogenicity prediction algorithms were used to estimate the reliability for the association of each variant with male infertility (Table 1 ). The conservation of the affected residues for each variant was also evaluated (Suppl. Fig. 6A).

Four of the TRIM71 missense variants identified in infertile men were also found in control subjects with complete spermatogenesis $(n=89)$ or in a Dutch cohort of proven fathers $(n=5784)$, making their association with male infertility rather unlikely (Fig 6E, green). In contrast, other identified TRIM71 
missense variants were present in one patient each but neither in control subjects nor in the Dutch cohort of proven fathers (Fig 6E, orange). Of those, c.368T>C/p.(Val123Ala), c.803T >A/p.(Leu268His), c.1070C $>$ T/p. (Ala357Val) and c.1486C >T/p.(Arg496Cys) were considered damaging by at least two out of four pathogenicity prediction algorithms (Table 1 ), and the respective residues for those variants were highly conserved among vertebrates (Suppl. Fig 6A). Thus, these variants have a higher likelihood of being associated with male infertility. As an additional line of evidence, all patients carrying variants in TRIM71 (Table 1) were evaluated for relevant variants in other genes previously reported in association with male infertility ( $\mathrm{n}=181$, listed in Suppl. Table 1). Importantly, one heterozygous frameshift variant was found in SYCP2 in patient M1686, who carries the TRIM71 variant c.1070C >T/p.(Ala357Val). SYCP2 was recently associated with male infertility ${ }^{57}$, and it is thus unlikely that this patient's TRIM71 variant alone is responsible for his cryptozoospermia, but an oligogenic cause of his phenotype could be hypothesized. Furthermore, a heterozygous missense variant for the gene NNT was identified in patient M1083, who carries the TRIM71 variant c.1486C >T/p.(Arg496Cys). In this case, the NNT missense variant is of unclear significance according to ACMG/AMP guidelines, and its functional contribution to the patients' phenotype can therefore neither be confirmed nor excluded at this point. Collectively, our analysis leaves the TRIM71 variants c.368T>C/p.(Val123Ala) and c.803T>A/p.(Leu268His) as the likeliest candidates for being associated with male infertility. Nevertheless, the functional effects of all TRIM71 missense variants should be evaluated experimentally in the future in order to definitively determine their pathogenicity.

\section{Exome sequencing data reveals an association of TRIM71 LoF variants with the SCO phenotype}

As mentioned above, a TRIM71 LoF variant - c.224_240dup/p.(Gly81CysfsTer24) - was identified via exome sequencing in subject M364, who presented with a clear SCO phenotype (Fig. 6E-F and Table 1). This variant consists of a duplication of 17 nucleotides which generates a frameshift resulting in a premature translation termination downstream of the RING domain (Suppl. Fig. 6B). This particular TRIM71 LoF variant has not been described in any public database so far, and was classified as likely pathogenic according to ACMG/AMP guidelines. Furthermore, the observed/expected (o/e) score computed for TRIM71 in the gnomAD database was extremely low (o/e score $=0.04,90 \%$ confidence interval 0.01-0.17). The o/e score compares the number of observed versus theoretically expected heterozygous LoF variants for a gene of interest. Without any selection pressure applied, an o/e ratio of around 1 would be expected for any given gene, whereas values below 0.35 are a rather clear sign of selection pressure against LoF variants, likely leading to haploinsufficiency intolerance.

Furthermore, subject M364 carries neither additional LoF variants nor likely pathogenic missense variants in any other known infertility-associated genes (listed in Suppl. Table 1). This patient presented with normal testicular volumes and LH levels, but increased FSH and decreased testosterone levels (Table 1), as is often the case in men with impaired spermatogenesis. Of note, no TRIM71 LoF variants were found in the Dutch cohort of proven fathers, and only one LoF variant was present in the exome data from 125,748 presumably healthy individuals from gnomAD ${ }^{58}$. This constitutes a significant enrichment of TRIM71 LoF variants in the MERGE cohort $(n=908 ; p=0.01)$ and an even higher enrichment in the SCO subcohort $(n=247 ; p=0.004)$, supporting a reliable association of TRIM71 LoF variants with human male infertility. 


\section{Discussion}

TRIM71 is a stem cell-specific protein expressed early in development and with an essential function for embryogenesis ${ }^{30-33}$. Embryonic lethality in Trim71 full knockout mice is accompanied by neural tube closure defects, revealing also a function of TRIM71 in the development of the nervous system ${ }^{30-33}$. Indeed, TRIM71 is known to promote self-renewal of neural progenitor cells ${ }^{21}$, and TRIM71 variants have been associated with human congenital hydrocephalus, a brain developmental disease characterized by enlarged brain ventricles due to an abnormal accumulation of cerebrospinal fluid ${ }^{59}$. Furthermore, TRIM71 has been connected with carcinogenesis in several studies ${ }^{22,24,29,55,60}$. Altogether, previous research on TRIM71 has been mostly focused on embryonic and neural stem cells as well as cancer cells. Our work here reports a novel role for TRIM71 in the generation and maintenance of germ cells during embryonic gonad development with crucial implications in murine and human fertility.

In line with previous studies ${ }^{33,34}$, our work reveals an expression of Trim71 in adult mouse testes which we found to be confined to SSCs (Fig. 1). We showed that germline-specific ablation of Trim71 early in mouse development causes a substantial reduction of gonad size and infertility in both sexes (Fig. 2). Further characterization of adult Trim 71 cKO mouse testes revealed a significant downregulation of SSCspecific and spermatid markers caused by a strong reduction in the number of developing germ cells (Fig. 2 ), a condition which in humans is known as Sertoli cell-only (SCO) phenotype ${ }^{43-45}$. Via exome sequencing of human infertile patients, we uncovered an association of TRIM71 with the SCO phenotype (Fig. 6).

In humans, infertility affects $10-15 \%$ of couples trying to conceive ${ }^{4}$. Male factors contribute in about half of couples and usually genetic causes correlate with a more severe spermatogenic impairment ${ }^{61}$. Lately, several genes have been identified as monogenic causes for azoospermia due to meiotic arrest (e.g.

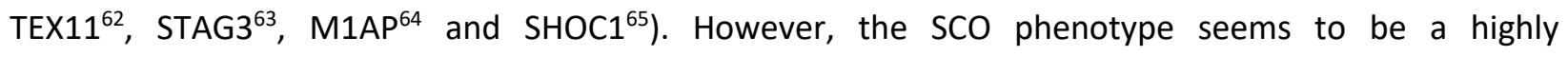
heterogeneous condition, as indicated by our data, making the identification of monogenic causes specially challenging. Our work uncovers TRIM71 as the first monogenic cause for this condition, based on the SCO-like phenotype observed in our germline-specific Trim71 cKO mouse together with the TRIM71 LoF variant that we found in a SCO patient. The finding of TRIM71 as a novel SCO candidate gene is relevant in terms of patient care, as it may allow future patients to be provided with a causal diagnosis for their azoospermia, and the potential success of testicular biopsy and sperm extraction with the aim to perform in vitro fertilization could be predicted in advance.

In contrast to the convincing relevance of the TRIM71 LoF variant, assessing the role of the identified rare TRIM71 missense variants in infertile patients of varying histological phenotypes is much more challenging. Of note, variants in the same gene may cause a spectrum of histological phenotypes ${ }^{64}$. Although our analysis provides a prediction for the pathogenicity of these variants, their functional effects on human male fertility are yet of unclear significance and can only be determined experimentally. Nevertheless, it is worth emphasizing that most missense variants identified in TRIM71 cluster outside of the NHL domain, as mutations affecting the $\mathrm{NHL}$ domain have been proven to be highly deleterious ${ }^{66,67}$ and compromise survival during early embryogenesis ${ }^{31}$. Future experiments using these TRIM71 variants should determine whether they functionally affect TRIM71's E3 ubiquitin ligase role, and if so, how exactly TRIM71-mediated ubiquitylation contributes to germ cell development. 
Our germline-specific Trim71 cKO mouse model provided further evidence for the role of Trim71 in germ cell development. The SCO-like phenotype was already apparent in the testes of neonatal (P0.5) cKO mice, indicating that TRIM71-induced germline defects have an embryonic origin (Fig. 3). However, early developmental defects are likely amplified during postnatal mitotic reactivation and pubertal spermatogenesis as described by a recent work showing infertility in a different germline-specific Trim 71 knockout (Trim $71^{-/ f l} ; D d \times 4^{\text {Cre/+ }}$ ) mouse mode ${ }^{34}$. In contrast to our observations in Trim $71^{f / /-} ;$ Nanos $3^{\text {Cre/+ }}$ mice, defects in Trim71 ${ }^{-/ f l} ; D d x 4^{C r e /+}$ mice were only detected in pubertal (P10) and adult (P56) mice, with germ cell numbers not yet altered in neonatal (P1) mice ${ }^{34}$. This discrepancy may result from an earlier Cre recombinase expression in our cKO model, since Nanos3 is expressed during PGC specification (E7.5) $)^{5,10,11}$, and $D d \times 4$ is expressed after colonization of the genital ridges (E10.5) ${ }^{14,15}$. In fact, Ddx4-induced recombination was previously reported to occur even after sex determination as late as E15.0 ${ }^{68}$. This might also explain why Trim $71^{\mathrm{fl} /-}$; $D d x 4^{\mathrm{Cre} / \mathrm{t}}$ females are fertile (Prof. Xin Wu, personal communication, June 22, 2020), while we found Trim $71^{\mathrm{fl} /-}$; Nanos $3^{\mathrm{Cre} / \mathrm{t}}$ females to be sterile. Notably, studies in C. elegans showed that the nematode homolog of TRIM71, LIN-41, is required for normal oocyte growth and meiotic maturation, with lin-41 depletion causing sterility in females ${ }^{69,70}$. Further studies are required to characterize the function of TRIM71 in mammalian female gonad development and fertility.

Although we do not exclude sex-specific functions of TRIM71 in the germline, we found both male and female Trim 71 cKO mice to be infertile, indicating that germ cell developmental defects may occur prior to sex determination of PGCs. Indeed, we observed PGC specification defects upon in-vitro differentiation of Trim71-deficient ESCs into PGCLCs, with reduced numbers of PGCLCs at the end of the specification process and decreased expression of the PGC-specific markers Prdm1/Blimp1, Nanos3 and SSEA-1/CD15 upon TRIM71 depletion (Fig. 4). Interestingly, Trim71 expression was still remarkably high in PGCLCs, suggesting its further involvement in subsequent stages of germ cell development. In fact, PGC specification defects may be followed by defects in the maintenance of the PGC pool during the stages of migration and genital ridge colonization, as we found that TRIM71 controls proliferation/self-renewal in the germline-derived tumor cell line NCCIT, which, to some extent, represent proliferative PGCs ${ }^{71}$.

The capability of self-renewal in a cell population reflects the balance between cell proliferation, differentiation and apoptosis. After PGC colonize the genital ridges, a significant expansion of the PGC pool occurs ${ }^{12,13,17,18}$. Furthermore, mitotically arrested male PGC undergo several massive apoptotic waves within the developing gonads: in mice, the first one occurs between E13.5 and E17.0 and is followed by a second one around birth and a third during the first wave of spermatogenesis between $\mathrm{P} 10$ and $\mathrm{P} 13^{72}$. Therefore, the role of TRIM71 in the maintenance of the PGC pool during these phases may extend beyond proliferation control, as previous studies have shown the involvement of TRIM71 not only in the control of cell proliferation, but also during cell differentiation and apoptosis. For instance, TRIM71 represses the mRNA of several cell cycle inhibitors, including CDKN1A, E2F7 and Rbp1/2, promoting proliferation of ESC and several cancer cell lines ${ }^{25,26,29}$. Proliferation of ESCs, neural progenitor cells, lung cancer cells and liver cancer cells was also promoted via TRIM71's E3 ubiquitin ligase function $21,22,55,73$. TRIM71 has also been shown to prevent apoptosis in developmental and oncogenic processes, specifically via p53 ubiquitylation and proteasomal degradation in breast cancer cells ${ }^{24}$ and ovarian cancer cells ${ }^{60}$ as well as in differentiating ESCs during mouse brain development ${ }^{23}$. More importantly, a recent study described an impaired proliferation and an increased apoptosis in primary murine SSCs upon TRIM71 knockdown ${ }^{34}$. Collectively, these findings together with our growth competition assays in NCCIT cells, support a role for TRIM71 in the control of proliferation and possibly also apoptosis. 
Finally, premature differentiation of TRIM71-deficient cells could be also contributing to the germ cell loss observed in our Trim71 cKO mice. A previous study has shown that Trim71-deficient ESCs are primed towards neural differentiation ${ }^{28}$. TRIM71 is also known to repress several mRNAs promoting differentiation, such as $H O X A 5^{26}$ or EGR $1^{27}$. Furthermore, Trim71-deficient ESCs showed a premature upregulation of differentiation-promoting micro-RNAs (miRNAs), including brain-specific and gonadspecific miRNAs ${ }^{28}$, highlighting a yet-unknown role for TRIM71-mediated miRNA regulation in early gonad development. Which mRNA, miRNA and/or protein targets are precisely regulated by TRIM71 to fulfill its functions in the germline remains to be further investigated.

In summary, we have shown that germline-specific Trim71 cKO male mice are infertile with testes predominantly displaying an SCO-like phenotype. We have identified TRIM71 variants in infertile men with severely impaired spermatogenesis, including a LoF variant in an SCO patient. The SCO-like phenotype was already apparent in neonatal P0.5 Trim71 cKO mice. Our in vitro assays suggested that germ cell deficiency in these mice may result from combined defects in the specification and maintenance of the PGC pool during embryonic gonad development. In a similar fashion, TRIM71 may support the maintenance of embryonic carcinoma cells and promote the development of GCT. Future unravelling of the molecular mechanisms by which TRIM71 governs germ cell biology will shed further light on the underlying causes of infertility. A deeper understanding of these processes will contribute to the development of new diagnostic and therapeutic strategies for reproductive medicine and for the treatment of GCT.

\section{Methods}

\section{Mouse generation}

All animal experiments were conducted in a licensed animal facility in accordance with the German law on the protection of experimental animals (the German animal welfare act), and were approved (approval number 87-51.04.2011.A063) by local authorities of the state of Nordrhein-Westfalen (Landesamt für Natur-, Umwelt- und Verbraucherschutz NRW).

The generation of the full Trim71 knockout mouse (Trim71flffl; Rosa26-CreERT2) was previously described $^{28}$. In order to generate a germline-specific Trim 71 knockout mouse, wild type females with floxed Trim71 alleles (WT, Trim $71^{f / f f}$ ) were bred with male mice expressing the Cre recombinase under the control of the endogenous Nanos3 promoter in heterozygosity ( $\left.\mathrm{Nanos}^{\mathrm{Cre} /+}\right)^{74}$. The heterozygous Trim $71^{-/+}$; Nanos $3^{\mathrm{Cre} /+}$ male offspring was then crossed with wild type Trim $71^{+/+}$females to produce Trim 71 wild type animals with the Nanos3-Cre allele in heterozygosity (WT, Trim $71^{+/+}$; Nanos $3^{\text {Cre } /+}$ ), or with wild type Trim $71^{f l / f l}$ females to produce germline-specific heterozygous (cHET, Trim $71^{-/+}$; Nanos $3^{\mathrm{Cr} / /+}$ ) and germline-specific knockout (cKO, Trim $71^{-/ f l}$; Nanos $^{\text {Cre/+ }}$ ) animals.

\section{Genomic DNA extraction and genotyping}

Genomic DNA was extracted from adult mice tail or ear biopsies or from mouse embryo yolk sacs by boiling the tissue in $50 \mathrm{mM} \mathrm{NaOH}$ for 20 minutes followed by $\mathrm{pH}$ neutralization of the lysate with 1/4 of 1 $\mathrm{M}$ Tris-Cl, $\mathrm{pH}$ 8.0. A three-primer strategy was used for PCR amplification of the Trim 71 locus or the Nanos3 locus and fragments were resolved on a $2 \%$ agarose gel. Primers used are listed in Suppl. Table 2. 


\section{Epidydmal sperm counts}

Cauda epididymis were isolated from 3-month-old male mice and transferred into 0.5-1 ml of PBS prewarmed at $35-37^{\circ} \mathrm{C}$. Sperm release was achieved by performing multiple cauda incisions followed by $10-$ min incubation at $35-37^{\circ} \mathrm{C}$ to allow the sperm swim out. The sperm suspension was then diluted in water (1:15-1:40) and sperm count was determined using a Neubauer hemocytometer.

\section{Isolation of spermatogonial stem cells from mouse testes}

Testes were isolated from 2-month-old male mice, and seminiferous tubules were exposed by removing the tunica albuginea. The tubules of several testes were pooled and incubated in approximately 10 volumes of HBSS with calcium and magnesium containing $1 \mathrm{mg} / \mathrm{ml}$ collagenase Type IV and 200 to $500 \mu \mathrm{g} / \mathrm{ml}$ DNAse I, followed by incubation at $37^{\circ} \mathrm{C}$ under gentle agitation for $15 \mathrm{~min}$. The tubules were then washed three times in 10 volumes of HBSS, followed by incubation at $37^{\circ} \mathrm{C}$ for 5 min in HBSS containing $0.25 \%$ trypsin and $150 \mu \mathrm{g} / \mathrm{ml}$ DNAse I under gentle agitation. Trypsinization was stopped by adding $20 \% \mathrm{FBS}$, and the cell suspension was filtered through a $40 \mu \mathrm{m}$ pore size nylon filter. The filtrate was centrifuged at $1000 \mathrm{rpm}$ for $5 \mathrm{~min}$ at $4{ }^{\circ} \mathrm{C}$ and the cell pellet was resuspended in PBS before cell counting. Magnetic-activated cell sorting (MACS) was used to enrich THY1.2+ cells. To this end, $10^{6}$ cells were stained with $0.2 \mu \mathrm{g}$ FITC-labelled anti-THY1.2/CD90.2 antibody (BioLegend) for $15 \mathrm{~min}$ at $4^{\circ} \mathrm{C}$. The cells were then washed in MACS-buffer (PBS supplemented with 2 mM EDTA and 0.5\% FBS) and incubated in a 1:5 dilution of anti-FITC microbeads (Miltenyi) for $15 \mathrm{~min}$ at $4{ }^{\circ} \mathrm{C}$ before a final washing in MACS buffer followed by centrifugation at $1000 \mathrm{rpm}$ for $5 \mathrm{~min}$ at $4{ }^{\circ} \mathrm{C}$. The pellet was resuspended in $500 \mu \mathrm{MACS}$ buffer and loaded on an AutoMACS device (Miltenyi) with the program set for positive selection. The efficiency of enrichment was 8-fold as later controlled by flow cytometry. The different cell populations were then used for RNA extraction and qRT-PCR quantification.

\section{H\&E staining of mouse testes cross-sections}

PFA-fixed paraffin-embedded murine testes cross-sections were deparaffinized by incubation at $65{ }^{\circ} \mathrm{C}$ for 15-20 min until the paraffin wax had melted, and washed in xylol twice for $10 \mathrm{~min}$. Next, the sections were rehydrated by a descending ethanol dilution series (100\% (2x), $95 \%, 90 \%, 80 \%, 70 \%)$ for 30 sec each and were kept in distilled water until staining. Cross-sections were stained in haematoxylin for 3 min and washed in cold running tap water for 3-5 min. Afterwards, the sections were counterstained with $0.5 \%$ eosin for $3 \mathrm{~min}$, removing excess dye by rinsing in cold running tap water for $1 \mathrm{~min}$. Cross-sections were then dehydrated in an ascending ethanol dilution series (70\%, $80 \%, 90 \%, 95 \%, 100 \%(2 x))$ and cleared in xylol twice for $2 \mathrm{~min}$. Paraffin sections were then mounted with the xylene-based DPX mounting media for histology (Sigma-Aldrich). H\&E stained sections were stored under the fume hood for $24 \mathrm{~h}$ before imaging by bright-field microscopy using the Zeiss Axio Lab.A1 microscope (Carl Zeiss).

\section{Immunofluorescence staining of testes cryosections}

PFA-fixed murine testis cryosections stored at $-20^{\circ} \mathrm{C}$ were defrosted and dried at room temperature for 15 min before their rehydration by washing in PBS and rinsing in distilled water. After drying the slides at RT for 10-15 min, cryosections were blocked (PBS supplemented with $1 \%$ BSA, $2 \%$ donkey serum and 0.3 $\%$ Triton X-100) for $1 \mathrm{~h}$ at RT while covered with parafilm to prevent evaporation. Primary antibodies diluted in PBST (0.3 \% Triton X-100/PBS) supplemented with $1 \%$ BSA were added and incubated at $4{ }^{\circ} \mathrm{C}$ overnight. On the next day, sections were washed three times for 5 min with PBST, and fluorescently- 
conjugated secondary antibodies diluted in PBST supplemented with $1 \%$ BSA, were added and incubated in the dark for $1 \mathrm{~h}$ at RT. The sections were then washed three times for 5 min with PBST and slides were mounted with Fluoromount-G containing DAPI and photobleaching inhibitors (SouthernBiotech). All steps were performed in a dark humid chamber to prevent evaporation and photobleaching. Stained sections were stored at $4{ }^{\circ} \mathrm{C}$ for $24 \mathrm{~h}$ prior to imaging by immunofluorescence microscopy. Images were taken using the Zeiss Observer.Z1 epifluorescence microscope (Carl Zeiss) and the ZEN 2012 (blue edition) software (Carl Zeiss). Antibodies used are listed in Suppl. Table 3.

\section{RNA extraction and qRT-PCR quantification}

RNA was extracted from cell pellets using the Trizol-containing reagent peqGold TriFAST according to the manufacturer's instructions (PeqLab). RNA pellets were resuspended in RNase-free water, and DNA digestion was performed prior to RNA quantification. 0.5-1 $\mu \mathrm{g}$ of RNA was reverse transcribed to cDNA using the High Capacity cDNA Reverse Transcription Kit (Applied Biosystems) according to the manufacturer's instructions. The cDNA was then diluted 1:5, and a relative quantification of specific genes was performed in a Bio-Rad qCycler using either TaqMan probes in iTaq Universal Probes Supermix or specific primer pairs in iTaq Universal SYBR Green Supermix (BioRad). Probes and Primers used are listed in Suppl. Table 2.

\section{Protein extraction and Western blotting}

Cell pellets were lyzed in RIPA buffer (20 mM Tris- $\mathrm{HCl}$ pH 7.5, $150 \mathrm{mM} \mathrm{NaCl}, 1 \mathrm{mM} \mathrm{Na}{ }_{2}$ EDTA, 1 mM EGTA, $1 \% \mathrm{NP}-40,1 \mathrm{mM} \mathrm{Na} \mathrm{VO}_{4}, 1 \%$ sodium deoxycholate, $2.5 \mathrm{mM}$ sodium pyrophosphate, $1 \mathrm{mM}$ glycerophosphate) supplemented with protease inhibitors and protein lysates were pre-cleared by centrifugation and quantified using the BCA assay kit (Pierce) according to the manufacturer's instructions. Protein lysates were then denatured by incubation with SDS buffer ( $12 \%$ glycerol, $60 \mathrm{mM}$ $\mathrm{Na}_{2}$ EDTA pH 8, $0.6 \%$ SDS, $0.003 \%$ bromophenol blue) for $10 \mathrm{~min}$ at $95^{\circ} \mathrm{C}$ and separated in SDS-PAGE gels in Laemmli buffer (25 mM Tris, $192 \mathrm{mM}$ glycine, $0.1 \%$ SDS). Proteins were then wet transferred to a nitrocellulose membrane in transfer buffer $(25 \mathrm{mM}$ Tris- $\mathrm{HCl} \mathrm{pH} 7.6,192 \mathrm{mM}$ glycine, 20\% methanol, $0.03 \%$ SDS) and membranes were blocked with $3 \% \mathrm{BSA}$ in $1 \times$ TBST $(50 \mathrm{mM}$ Tris- $\mathrm{HCl} \mathrm{pH} \mathrm{7.6,150} \mathrm{mM} \mathrm{NaCl}$, $0.05 \%$ Tween-20) prior to overnight incubation at $4{ }^{\circ} \mathrm{C}$ with the required primary antibodies. After washing the membrane three times with $1 \times$ TBST, they were incubated with a suitable HRP-coupled secondary antibody for $1 \mathrm{~h}$ at RT, followed by three washing steps with $1 \times$ TBST. Membranes were developed with the ECL substrate kit (Pierce) according to the manufacturer's instructions. Antibodies used are listed in Suppl. Table 3.

\section{Cell culture}

Derivation of wild type murine ESCs (WT, Trim71 fl/fl) from conditional Trim 71 full knockout mice (Trim71 $1^{f / f l}$; Rosa26-CreERT2) was previously described ${ }^{28}$. Trim 71 knockout murine ESCs (KO, Trim $71^{\%}$ ) were generated from wild type ESCs by addition of $500 \mathrm{nM}$ of 4-hydroxytamoxifen in their culture media for $48 \mathrm{~h}$, followed by further culture for $72 \mathrm{~h}$ to achieve full protein depletion. ESCs were cultured in 0.1 $\%$ gelatin-coated dishes and maintained in 2i+LIF media (DMEM knockout media supplemented with 15 \% FCS, 1 \% penicillin-streptomycin, 0.1 mM NEAA, 2 mM L-GlutaMAX, $100 \mu \mathrm{M} \beta$ - mercaptoethanol, $0.2 \%$ in-house produced LIF, $1 \mu \mathrm{M}$ of MEK/ERK inhibitor PD0325091 and $3 \mu \mathrm{M}$ of GSK-3 inhibitor CHIR99021). 
The human hepatocellular carcinoma cell line HepG2 and the human embryonic carcinoma cell lines JKT1, NCCIT, NTERA-2, TCam-2 and 2102EP were acquired from ATCC. HepG2, NCCIT and TCam-2 were cultured in RPMI 1640 media supplemented with 10\% FBS and 1\% penicillin-streptomycin antibiotic solution. JKT-1, NTERA -2 and 2012EP cells were cultured in DMEM media supplemented with $10 \%$ FBS and $1 \%$ penicillin- streptomycin antibiotic solution.

\section{In vitro differentiation of ESCs into PGCLCs}

ESCs growing under naïve pluripotency $(\mathrm{d} 0$ ) conditions (2i+LIF media on $0.1 \%$ gelatine-coated dishes) were primed to EpiSCs (resembling post-implantation epiblast-derived stem cells) for two days by plating $10^{5}$ cells per well on 12-well plates coated with $20 \mu \mathrm{g} / \mathrm{ml}$ fibronectin in priming media (N2B27 media supplemented with $1 \%$ penicillin-streptomycin, $2 \mathrm{mM} \mathrm{L-GlutaMAX}, 1 \% \mathrm{KSR}, 20 \mathrm{ng} / \mathrm{ml}$ Activin A and $12 \mathrm{ng} / \mathrm{ml}$ bFGF). After $24 \mathrm{~h}$ (d1), fresh priming media was provided. After $48 \mathrm{~h}$ (d2), EpiSCs were detached with Accutase Stem Cell Pro and 5000 cells per well were plated on a suspension U-bottom 96-well dish in specification media (GMEM media supplemented with $15 \% \mathrm{KSR}, 1 \%$ penicillin-streptomycin, $0.1 \mathrm{mM}$ NEAA, 2 mM L-GlutaMAX, $100 \mu \mathrm{M} \beta$-mercaptoethanol, $1 \mathrm{mM}$ sodium pyruvate, $0.2 \%$ in-house produced LIF, $50 \mathrm{ng} / \mathrm{ml} \mathrm{EGF,} 100 \mathrm{ng} / \mathrm{ml} \mathrm{SCF}$ and $500 \mathrm{ng} / \mathrm{ml} \mathrm{BMP4).} \mathrm{After} 6$ days in this media (d8), cells growing in spheroids were recovered, detached by trypsinization for $10 \mathrm{~min}$ at $37^{\circ} \mathrm{C}$ and either used for RNA extraction or double-stained with anti-ITGB3/CD61 and anti-SSEA-1/CD15 antibodies to determine the number of PGCLCs by flow cytometry. For staining, trypsinized cells were washed in $0.1 \%$ BSA/PBS, incubated with the antibodies diluted in $0.1 \% \mathrm{BSA} / \mathrm{PBS}$ for $15 \mathrm{~min}$ at $4{ }^{\circ} \mathrm{C}$ in the dark and washed again in $0.1 \% \mathrm{BSA} / \mathrm{PBS}$. Isotype control antibodies were used to control single stainings (data not shown). These protocols were adapted from a previous work ${ }^{46}$. Antibodies used are listed in Suppl. Table 3.

\section{Generation of NCCIT cells with TRIM71 frameshift mutations}

Gene editing using the CRISPR/Cas9 system was carried out using the plasmid pSpCas9(BB)-2A-GFP (PX458), which was a kind gift from Feng Zhang (Addgene plasmid \#48138), after the insertion of specific sgRNAs targeting TRIM71 RING (sgRNA $\triangle$ RING 5'-CACCGCTCGCAGACGCTCACGCTGT-3') or NHL (sgRNA $\triangle$ NHL6 5'-CACCGCACAACGATCATTCCGCTGG-3') domains. NCCIT cells were transfected with PX458 empty vector (EV), TRIM71 $\triangle$ RING vector or TRIM71 $\triangle$ NHL6 vector using Lipofectamine Stem Transfection Reagent (Invitrogen) following the manufacturer's instructions. $48 \mathrm{~h}$ post- transfection, transfected (GFPpositive) cells were sorted by FACS and plated as a bulk population. Growth competition assays (see below) were performed with sorted bulk populations. Generation of single clones from the bulk populations was also performed for subsequent Sanger sequencing and Western blot analysis.

\section{Growth competition assays and NGS allele frequency analysis via Illumina MiSeq}

In order to analyze the growth behavior of CRISPR/Cas9-edited NCCIT cells, GFP-positive sorted bulk EVtransfected cells (WT) were mixed 1:1 with either TRIM71 sgRNA $\triangle$ RING-transfected cells or TRIM71 sgRNA $\triangle$ NHL6-transfected cells (d0) and cultured ( $2 \times 10^{5}$ cells per well in a 6-well plate) for three weeks to investigate the population dynamics. Samples $\left(2 \times 10^{5}\right.$ cells) were taken at $d 0, d 3, d 7, d 14$ and d21 for genomic DNA extraction. A double PCR strategy was then applied for the preparation of barcoded amplicons. In a first PCR reaction, the region around the CRISPR/Cas9-targeted site was amplified using site-specific forward and reverse primers with a common $5^{\prime}$ overhang. After PCR product cleaning, a second PCR was conducted with Illumina index primers which bind to the common 5 'overhangs of the first primers and also contain unique barcode/index sequences followed by specific 5' (P5) or 3' (P7) 
adaptor sequences. PCR products were then gel-purified and sequenced on an Illumina MiSeq sequencing platform via amplification with common primers binding to P5/P7 adaptor sequences. Data analysis was performed using CRISPResso $2^{75}$ with the quality cut-off set at 30 and the minimum identity score for the alignment being adjusted to 50 . After analysis of the sequencing data, single reads were categorized as wild type, frameshift mutations and in-frame mutations, and allele frequencies were calculated and displayed as percentages in a pie chart. Comparing changes in the distribution of single reads for wild type (EV) and frameshift mutants TRIM71 $\triangle$ RING or TRIM71 $\triangle$ NHL6 over time gives insights into the dynamics of each cell populations within the initially 1:1 mixed culture. Allele frequencies of pure wild type (EV) were also analyzed at $\mathrm{d} 0$ and $\mathrm{d} 21$ as a control. Analysis of allele frequencies of pure WT, TRIM71 $\triangle \mathrm{RING}$ and TRIM71 $\triangle$ NHL6 populations before (d0 pure) and after mixing them 1:1 (d0 1:1) ensured that no PCR product was biased over another based on theoretically expected vs. observed/measured allele frequencies.

\section{Exome sequencing study population}

The study population consisted of 1025 individuals (MERGE cohort), of whom 908 otherwise healthy men presented with quantitative spermatogenic impairment. Patients attended the Centre of Reproductive Medicine and Andrology (CeRA) at the University Hospital Münster or the Clinic for Urology, Pediatric Urology and Andrology in Gießen. A subgroup of 247 selected patients was used to investigate novel genetic causes of the SCO phenotype (SCO subcohort). They had previously undergone testicular biopsy and presented with complete and bilateral absence of germ cells. In addition to this subgroup, 89 male subjects with complete spermatogenesis also pertaining to the MERGE cohort were included in the study as controls. All individuals gave written informed consent and the study protocol has been approved by the appropriate ethics committees according to the Declaration of Helsinki (Münster: Kennzeichen 2010578-f-S, Gießen: No. 26/11). As further controls, we utilized a Dutch cohort of 5784 proven fathers sequenced at the Radboudumc genome diagnostics centre in Nijmegen, the Netherlands. These were healthy fathers of a child with severe developmental delay and all parents underwent routine exome sequencing for trio analysis. The fertility of the fathers is expected to be similar to an unselected sample of the population.

\section{Exome sequencing data generation and analysis}

In order to perform exome sequencing, genomic DNA was isolated from the subjects' peripheral blood applying standard procedures. Target enrichment, library capture, exome sequencing and variant calling were performed as previously described ${ }^{64}$. Briefly, DNA was enriched according to Agilent's SureSelect ${ }^{\text {QXT }}$ Target Enrichment for Illumina Multiplexed Sequencing Featuring Transposase-Based Library Prep Technology or Twist Bioscience's Twist Human Core Exome protocol. For library capture, SureSelectXT Human All Exon Kits (Agilent) or Human Core Exome (Twist Bioscience) were used. Sequencing was then

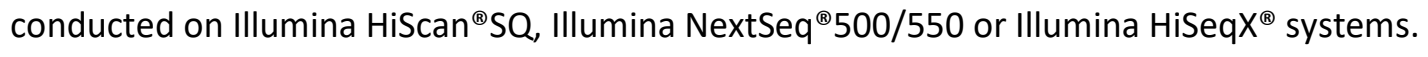

Exome data analysis was conducted on 247 SCO patients using our platform Sciobase. We filtered genes based on the predicted effect of the variant on translated protein (including only stop-gain, frameshift, and splice donor/acceptor variants) and the allele frequency in the general population (including only rare variants (MAF $\leq 0.01$ ) as listed in gnomAD genomic database (v2.1.1: https://gnomad.broadinstitute.org)). This first filtering step resulted in the selection of 3997 genes. We then developed an application termed Haystack to facilitate the search for previously unknown causes of genetic diseases, technically applicable 
to exome data from patients with any clinical phenotype. In brief, Haystack aggregates information from multiple databases in an R Shiny application and allows for filtering based on the aggregated data, thus minimizing the manual effort required for candidate gene selection. All source code is publicly available at GitHub (https://github.com/MarWoes/haystack). We used Haystack to filter variants from our SCO patients for quality (read depth $\geq 10$, variant depth $\geq 4$ and frequency $\geq 40 \%$ ). The remaining 2870 genes were filtered again based on genotype and allele frequency in gnomAD (MAF $\leq 0.01$ for homozygous variants and MAF $\leq 0.001$ for heterozygous and X-linked variants) as well as on expression levels in the testes (including only genes whose expression in testis was above $50 \%$ of its maximum expression across all tissues according to the GTEx portal (v7: https://gtexportal.org)). Last, genes with LoF variants present in individuals with intact spermatogenesis were excluded from the selected 949 genes, leaving a total of 721 genes with LoF variants specifically found in SCO patients. TRIM71 was found among these genes in a patient with an SCO phenotype (subject M364). This individual's DNA was subjected to Sanger sequencing according to standard procedures in order to confirm his TRIM71 frameshift variant. PCR was performed using the primers: F: 5'-TGCAGGCCTAATCGATGCAT-3'; R: 5'-GAAGAAGCTGCGTTGCCCTC-3'.

For the statistical analysis of exome data, the number of alleles affected and unaffected by LoF variants in TRIM71 in all gnomAD subjects was compared to affected and unaffected alleles in the 908 patients with quantitative spermatogenic impairment (MERGE cohort) and the 247 SCO patients (SCO subcohort). A two-sided Fisher's exact test was performed using the Woolf logit method.

In a subsequent step, exome data of the entire MERGE cohort and the Dutch father cohort was screened for rare (see MAF cutoffs above) missense variants in TRIM71 (NM_001039111). The Z score for TRIM71 was obtained from gnomAD database. Predicted pathogenicity was assessed using the scoring algorithms CADD (v1: https://cadd.gs.washington.edu/), PolyPhen (v2: http://genetics.bwh.harvard.edu/pph2), SIFT (v2: https://sift.bii.a-star.edu.sg), and Mutation Taster (v6: http://www.mutationtaster.org). Known genetic causes of azoospermia, such as chromosomal aberrations and AZF deletions were excluded in all infertile patients with LoF or missense variants in TRIM71. Furthermore, all patients carrying LoF or missense variants in TRIM71 (listed in Table 1) were evaluated for relevant variants in other genes ( $\mathrm{n}=181$, Suppl. Table 1) that could explain the patients' infertility. The majority of examined genes ( $n=170$, Suppl. Table $1 \mathrm{~A}$ ) were taken from a review on the genetics of male infertility ${ }^{76}$. The genes were selected based on associated phenotypes and had been rated with limited to definitive clinical evidence. The list was amended by adding 11 recently published genes (Suppl. Table 1B). Specifically, the patients' exome data was screened for stop-gain, frameshift, and splice acceptor/donor variants as well as missense variants with a CADD score $\geq 20$. Only rare variants (gnomAD MAF $\leq 0.01$ for homozygous and MAF $\leq 0.001$ for heterozygous variants) were taken into account. For recessive genes, variants were only considered if found in homozygous or likely compound-heterozygous state (two variants in the same gene in the same patient).

\section{Acknowledgements}

We thank all members of the Kolanus lab and the LIMES institute for general advice and discussion. LTF held a stipend from Bayer AG. WK is funded by the Deutsche Forschungsgemeinschaft (DFG, German Research Foundation) under Germany's Excellence Strategy EXC2151 - 390873048. We are indebted to all subjects having donated their DNA for research use in our studies. The technical work of Christina 
Burhöi and Judith Ryll is gratefully acknowledged. This work was carried out within the frame of the German Research Foundation (DFG) funded Clinical Research Unit 'Male Germ Cells: from Genes to Function' (CRU326, grants to NN, CF and FT).

\section{Author contributions}

LTF, JE, YP and SM designed and performed experiments. LTF and JE wrote the manuscript. SM and SS developed the germline-specific Trim71 KO mouse. YP, SM, SS and LTF performed in vivo and in vitro experiments. MH and YP performed NGS Illumina MiSeq. NN and SDP provided testis single-cell RNAseq data. SK and DF provided samples and histological evaluation for patients from Münster and Gießen, respectively. JE and MW developed the variant filtering tool Haystack. JE performed bioinformatics analyses. MO provided genetic data for the cohort of Dutch fathers. WK, FT, HS and CF supervised experimental design, data analysis and manuscript writing.

\section{References}

1. Agarwal, A., Mulgund, A., Hamada, A. \& Chyatte, M. R. (2015). A unique view on male infertility around the globe. Reprod. Biol. Endocrinol. 13, 37

2. Raman, J. D., Nobert, C. F. \& Goldstein, M. (2005). Increased incidence of testicular cancer in men presenting with infertility and abnormal semen analysis. J. Urol. 174, 1819-1822

3. Looijenga, L. H. J., Hersmus, R., De Leeuw, B. H. C. G. M., Stoop, H., Cools, M., Oosterhuis, J. W., Drop, S. L. S. \& Wolffenbuttel, K. P. (2010). Gonadal tumours and DSD. Best Pract. Res. Clin. Endocrinol. Metab. 24, 291-310

4. Zorrilla, M. \& Yatsenko, A. N. (2013). The Genetics of Infertility: Current Status of the Field. Curr. Genet. Med. Rep. 1, 247-260

5. Lawson, K. A., Dunn, N. R., Roelen, B. A. J., Zeinstra, L. M., Davis, A. M., Wright, C. V. E., Korving, J. P. W. F. M. \& Hogan, B. L. M. (1999). Bmp4 is required for the generation of primordial germ cells in the mouse embryo. Genes Dev. 13, 424-436

6. Yamaji, M., Seki, Y., Kurimoto, K., Yabuta, Y., Yuasa, M., Shigeta, M., Yamanaka, K., Ohinata, Y. \& Saitou, M. (2008). Critical function of Prdm14 for the establishment of the germ cell lineage in mice. Nat. Genet. 40, 1016-1022

7. Ohinata, Y. et al. (2005). Blimp1 is a critical determinant of the germ cell lineage in mice. Nature 436, 207-213

8. Kurimoto, K., Yabuta, Y., Ohinata, Y., Shigeta, M., Yamanaka, K. \& Saitou, M. (2008). Complex genome-wide transcription dynamics orchestrated by Blimp1 for the specification of the germ cell lineage in mice. Genes Dev. 22, 1617-1635

9. Wang, J.-Q. \& Cao, W.-G. (2016). Key Signaling Events for Committing Mouse Pluripotent Stem Cells to the Germline Fate1. Biol. Reprod. 94(1):24

10. Ginsburg, M., Snow, M. H. L. \& Mclaren, A. (1990). Primordial germ cells in the mouse embryo during gastrulation. Development 110(2):521-8 
11. Tsuda, M., Sasaoka, Y., Kiso, M., Abe, K., Haraguchi, S., Kobayashi, S. \& Saga, Y. (2003). Conserved role of nanos proteins in germ cell development. Science (80-. ). 301, 1239-1241

12. Anderson, R., Copeland, T. K., Schöler, H., Heasman, J. \& Wylie, C. (2000). The onset of germ cell migration in the mouse embryo. Mech. Dev. 91, 61-68

13. Tam, P. P. L. \& Snow, M. H. L. (1981). Proliferation and migration of primordial germ cells during compensatory growth in mouse embryos. J. Embryol. Exp. Morphol. Vol. 64, 133-147

14. Fujiwara, Y., Komiya, T., Kawabata, H., Satot, M., Fujimotot, H., Furusawa, M. \& Nocet, T. (1994). Isolation of a DEAD-family protein gene that encodes a murine homolog of Drosophila vasa and its specific expression in germ cell lineage. Proc. Natl. Acad. Sci. U.S.A. 91(25):12258-62

15. Tanaka, S. S., Toyooka, Y., Akasu, R., Katoh-Fukui, Y., Nakahara, Y., Suzuki, R., Yokoyama, M. \& Noce, T. (2000). The mouse homolog of Drosophila Vasa is required for the development of male germ cells. Genes Dev. 14, 841-853

16. Enders, G. C. \& May, J. J. (1994). Developmentally regulated expression of a mouse germ cell nuclear antigen examined from embryonic day 11 to adult in male and female mice. Dev. Biol. 163, 331-340

17. Ewen, K. A. \& Koopman, P. (2010). Mouse germ cell development: From specification to sex determination. Molecular and Cellular Endocrinology 323, 76-93

18. Bowles, J. \& Koopman, P. (2010). Sex determination in mammalian germ cells: Extrinsic versus intrinsic factors. Reproduction 139, 943-958

19. Pierce, J. L., Frazier, A. L. \& Amatruda, J. F. (2018). Pediatric Germ Cell Tumors: A Developmental Perspective. Advances in Urology 2018:9059382

20. Ecsedi, M. \& Grosshans, H. (2013). LIN-41/TRIM71: emancipation of a miRNA target. Genes Dev. 27, 581-9

21. Chen, J., Lai, F. \& Niswander, L. (2012). The ubiquitin ligase mLin41 temporally promotes neural progenitor cell maintenance through FGF signaling. Genes Dev. 26(8):803-15

22. Ren, H. et al. (2018). E3 ubiquitin ligase tripartite motif-containing 71 promotes the proliferation of non-small cell lung cancer through the inhibitor of kappaB- $\alpha$ /nuclear factor kappaB pathway. Oncotarget 9, 10880-90

23. Nguyen, D. T. T., Richter, D., Michel, G., Mitschka, S., Kolanus, W., Cuevas, E. \& Wulczyn, F. G. (2017). The ubiquitin ligase LIN41/TRIM71 targets p53 to antagonize cell death and differentiation pathways during stem cell differentiation. Cell Death Differ. 24, 1063-78

24. Hu, Q. et al. (2019). Oncogenic IncRNA downregulates cancer cell antigen presentation and intrinsic tumor suppression. Nat. Immunol. 20, 835-51

25. Chang, H.-M., Martinez, N. J., Thornton, J. E., Hagan, J. P., Nguyen, K. D. \& Gregory, R. I. (2012). Trim71 cooperates with microRNAs to repress Cdkn1a expression and promote embryonic stem cell proliferation. Nat. Commun. 3, 923

26. Loedige, I., Gaidatzis, D., Sack, R., Meister, G. \& Filipowicz, W. (2013). The mammalian TRIM-NHL protein TRIM71/LIN-41 is a repressor of mRNA function. Nucleic Acids Res. 41, 518-32 
27. Worringer, K. A., Rand, T. A., Hayashi, Y., Sami, S., Takahashi, K., Tanabe, K., Narita, M., Srivastava, D. \& Yamanaka, S. (2014). The let-7/LIN-41 pathway regulates reprogramming to human induced pluripotent stem cells by controlling expression of prodifferentiation genes. Cell Stem Cell 14, 4052

28. Mitschka, S. et al. (2015). Co-existence of intact stemness and priming of neural differentiation programs in mES cells lacking Trim71. Sci. Rep. 5, 11126

29. Torres-Fernández, L. A., Jux, B., Bille, M., Port, Y., Schneider, K., Geyer, M., Mayer, G. \& Kolanus, W. (2019). The mRNA repressor TRIM71 cooperates with Nonsense-Mediated Decay factors to destabilize the mRNA of CDKN1A/p21. Nucleic Acids Res. 47(22):11861-79

30. Schulman, B. R. M., Esquela-Kerscher, A. \& Slack, F. J. (2005). Reciprocal expression of lin-41 and the microRNAs let-7 and mir-125 during mouse embryogenesis. Dev. Dyn. 234, 1046-54

31. Schulman, B. R. M., Liang, X., Stahlhut, C., Delconte, C., Stefani, G. \& Slack, F. J (2008). The let-7 microRNA target gene, Mlin41/Trim71 is required for mouse embryonic survival and neural tube closure. Cell Cycle. 7(24):3935-42

32. Cuevas, E., Rybak-Wolf, A., Rohde, A. M., Nguyen, D. T. T. \& Wulczyn, F. G. (2015). Lin41/Trim71 is essential for mouse development and specifically expressed in postnatal ependymal cells of the brain. Front. Cell Dev. Biol. 3:20

33. Rybak, A., Fuchs, H., Hadian, K., Smirnova, L., Wulczyn, E. A., Michel, G., Nitsch, R., Krappmann, D. \& Wulczyn, F. G. (2009). The let-7 target gene mouse lin-41 is a stem cell specific E3 ubiquitin ligase for the miRNA pathway protein Ago2. Nat. Cell Biol. 11, 1411-20

34. Du, G. et al. (2020). mRBPome capture identifies the RNA binding protein TRIM71, an essential regulator of spermatogonial differentiation. Development dev. 147(8):184655

35. Liao, H.-F., Kuo, J., Lin, H.-H. \& Lin, S.-P. (2016). Isolation of THY1+ Undifferentiated Spermatogonia from Mouse Postnatal Testes Using Magnetic-activated Cell Sorting (MACS). Bio-protocol 6(24): e2072.

36. Gassei, K. \& Orwig, K. E. (2013). SALL4 Expression in Gonocytes and Spermatogonial Clones of Postnatal Mouse Testes. PLoS One 8, e53976

37. Zheng, K., Wu, X., Kaestner, K. H. \& Wang, P. J. (2009). The pluripotency factor LIN28 marks undifferentiated spermatogonia in mouse. BMC Dev. Biol. 9,38

38. Gaytan, F. et al. (2013). Distinct expression patterns predict differential roles of the mirna-binding proteins, lin28 and lin28b, in the mouse testis: Studies during postnatal development and in a model of hypogonadotropic hypogonadism. Endocrinology 154, 1321-36

39. Hermann, B. P. et al. (2018). The Mammalian Spermatogenesis Single-Cell Transcriptome, from Spermatogonial Stem Cells to Spermatids. Cell Rep. 25, 1650-67

40. Koshimizu, U., Nishioka, H., Watanabe, D., Dohmae, K. \& Nishimune, Y. (1995). Characterization of a novel spermatogenic cell antigen specific for early stages of germ cells in mouse testis. Mol. Reprod. Dev. 40, 221-27

41. Tanaka, H., Pereira, L. A. V. D., Nozaki, M., Tsuchida, J., Sawada, K., Mori, H. \& Nishimune, Y. (1998). A germ cell-specific nuclear antigen recognized by a monoclonal antibody raised against mouse 
testicular germ cells. Int. J. Androl. 20, 361-66

42. Mundlos, S., Pelletier, J., Darveau, A., Bachmann, M., Winterpacht, A. \& Zabel, B. (1993). Nuclear localization of the protein encoded by the Wilms' tumor gene WT1 in embryonic and adult tissues. Development 119, 1329-41

43. Tüttelmann, F., Simoni, M., Kliesch, S., Ledig, S., Dworniczak, B., Wieacker, P. \& Röpke, A. (2011). Copy number variants in patients with severe oligozoospermia and sertoli-cell-only syndrome. PLoS One 6(4):e19426

44. Tüttelmann, F., Ruckert, C. \& Röpke, A. (2018). Disorders of spermatogenesis. Medizinische Genet. 30, $12-20$

45. Koc, G., Ozdemir, A. A., Girgin, G., Akbal, C., Kirac, D., Avcilar, T. \& Guney, A. I. (2019). Male infertility in Sertoli cell-only syndrome: An investigation of autosomal gene defects. Int. J. Urol. 26, 292-298

46. Hayashi, K., Ohta, H., Kurimoto, K., Aramaki, S. \& Saitou, M. (2011). Reconstitution of the mouse germ cell specification pathway in culture by pluripotent stem cells. Cell 146, 519-532

47. Guo, G., Yang, J., Nichols, J., Hall, J. S., Eyres, I., Mansfield, W. \& Smith, A. (2009). Klf4 reverts developmentally programmed restriction of ground state pluripotency. Development 136, 106369

48. Veillard, A. C. et al. (2014). Stable methylation at promoters distinguishes epiblast stem cells from embryonic stem cells and the in vivo epiblasts. Stem Cells Dev. 23, 2014-29

49. Grabole, N., Tischler, J., Hackett, J. A., Kim, S., Tang, F., Leitch, H. G., Magnúsdóttir, E. \& Surani, M. A. (2013). Prdm14 promotes germline fate and naive pluripotency by repressing FGF signalling and DNA methylation. EMBO Rep. 14, 629-37

50. Seki, Y. (2018). PRDM14 is a unique epigenetic regulator stabilizing transcriptional networks for pluripotency. Frontiers in Cell and Developmental Biology 6,12

51. Kehler, J. et al. (2004). Oct4 is required for primordial germ cell survival. EMBO Rep. 5, 1078-83

52. Rosner, M. H., Vigano, M. A., Ozato, K., Timmons, P. M., Poirie, F., Rigby, P. W. J. \& Staudt, L. M. (1990). A POU-domain transcription factor in early stem cells and germ cells of the mammalian embryo. Nature 345, 686-92

53. Tang, Z., Li, C., Kang, B., Gao, G., Li, C. \& Zhang, Z. (2017). GEPIA: A web server for cancer and normal gene expression profiling and interactive analyses. Nucleic Acids Res. 45, W98-W102

54. Ravi, R. K., Walton, K. \& Khosroheidari, M. (Humana Press Inc., 2018). Miseq: A next generation sequencing platform for genomic analysis. Methods in Molecular Biology 1706, 223-32

55. Chen, Y. L., Yuan, R. H., Yang, W. C., Hsu, H. C. \& Jeng, Y. M. (2013). The stem cell E3-ligase Lin-41 promotes liver cancer progression through inhibition of microRNA-mediated gene silencing. $J$. Pathol. 229, 486-496

56. Wiel, L., Baakman, C., Gilissen, D., Veltman, J. A., Vriend, G. \& Gilissen, C. (2019). MetaDome: Pathogenicity analysis of genetic variants through aggregation of homologous human protein domains. Hum. Mutat. 40, 23798 
57. Schilit, S. L. P. et al. (2019). SYCP2 Translocation-Mediated Dysregulation and Frameshift Variants Cause Human Male Infertility. Am. J. Hum. Genet. 106, 41-57

58. Karczewski, K. J. et al. (2020). The mutational constraint spectrum quantified from variation in 141,456 humans. Nature $\mathbf{5 8 1}, 434-43$

59. Furey, C. G. et al. (2018). De Novo Mutation in Genes Regulating Neural Stem Cell Fate in Human Congenital Hydrocephalus. Neuron 99, 302-14

60. Chen, Y., Hao, Q., Wang, J., Li, J., Huang, C., Zhang, Y., Wu, X., Lu, H. \& Zhou, X. (2019). Ubiquitin ligase TRIM71 suppresses ovarian tumorigenesis by degrading mutant p53. Cell Death Dis. 10, 114

61. Lee, J. Y., Dada, R., Sabanegh, E., Carpi, A. \& Agarwal, A. (2011). Role of genetics in azoospermia. Urology 77, 598-601

62. Yatsenko, A. N. et al. (2015). X-linked TEX11 mutations, meiotic arrest, and azoospermia in infertile men. N. Engl. J. Med. 372, 2097-107

63. Van Der Bijl, N., Röpke, A., Biswas, U., Wöste, M., Jessberger, R., Kliesch, S., Friedrich, C. \& Tüttelmann, F. (2019). Mutations in the stromal antigen 3 (STAG3) gene cause male infertility due to meiotic arrest. Hum. Reprod. 34, 2112-19

64. Wyrwoll, M. J. et al. (2020). Bi-allelic Mutations in M1AP Are a Frequent Cause of Meiotic Arrest and Severely Impaired Spermatogenesis Leading to Male Infertility. Am. J. Hum. Genet. 107(2): 342-51

65. Krausz, C. et al. (2020). Genetic dissection of spermatogenic arrest through exome analysis: clinical implications for the management of azoospermic men. Genet. Med. doi: 10.1038/s41436-0200907-1.

66. Slack, F. J., Basson, M., Liu, Z., Ambros, V., Horvitz, H. R. \& Ruvkun, G. (2000). The lin-41 RBCC gene acts in the $C$. elegans heterochronic pathway between the let-7 regulatory RNA and the LIN-29 transcription factor. Mol. Cell 5, 659-69

67. Kumari, P. et al. (2018). Evolutionary plasticity of the NHL domain underlies distinct solutions to RNA recognition. Nat. Commun. 9, 1549

68. Gallardo, T., Shirley, L., John, G. B. \& Castrillon, D. H. (2007). Generation of a germ cell-specific mouse transgenic Cre line, Vasa-Cre. Genesis 45, 413-417

69. Spike, C. A., Coetzee, D., Eichten, C., Wang, X., Hansen, D. \& Greenstein, D. (2014). The TRIM-NHL protein LIN-41 and the OMA RNA-binding proteins antagonistically control the prophase-tometaphase transition and growth of caenorhabditis elegans oocytes. Genetics 198, 1535-58

70. Tsukamoto, T., Gearhart, M. D., Spike, C. A., Huelgas-Morales, G., Mews, M., Boag, P. R., Beilharz, T. H. \& Greenstein, D. (2017). LIN-41 and OMA Ribonucleoprotein Complexes Mediate a Translational Repression-to-Activation Switch Controlling Oocyte Meiotic Maturation and the Oocyte-to-Embryo Transition in Caenorhabditis elegans. Genetics 206, 2007-39

71. Nettersheim, D., Jostes, S., Schneider, S. \& Schorle, H. (2016). Elucidating human male germ cell development by studying germ cell cancer. Reproduction 152, R101-13

72. Wang, R. A., Nakane, P. K. \& Koji, T. (1998). Autonomous cell death of mouse male germ cells during 
fetal and postnatal period. Biol. Reprod. 58, 1250-56

73. Li, Y. P. et al. (2019). A TRIM71 binding long noncoding RNA Trincr1 represses FGF/ERK signaling in embryonic stem cells. Nat. Commun. 10(1):1368.

74. Suzuki, H., Tsuda, M., Kiso, M. \& Saga, Y. (2008). Nanos3 maintains the germ cell lineage in the mouse by suppressing both Bax-dependent and -independent apoptotic pathways. Dev. Biol. 318, 133-142

75. Pinello, L., Canver, M. C., Hoban, M. D., Orkin, S. H., Kohn, D. B., Bauer, D. E. \& Yuan, G. C. (2016). Analyzing CRISPR genome-editing experiments with CRISPResso. Nature Biotechnology 34, 695-97

76. Oud, M. S., Volozonoka, L., Smits, R. M., Vissers, L. E. L. M., Ramos, L. \& Veltman, J. A. (2019). A systematic review and standardized clinical validity assessment of male infertility genes. Hum. Reprod. 34, 932-41

Table 1: TRIM71 variants identified in infertile men within the MERGE cohort.

aPredicted effect of each TRIM71 variant estimated by four different pathogenicity prediction algorithms (CADD/PolyPhen2/SIFT/MutationTaster, shown in the same order). For CADD, variants with values above 20 are more likely to be deleterious to protein function. For the rest, $\mathrm{D}=$ damaging/deleterious; $T=$ tolerated; $\mathrm{B}=$ benign; $\mathrm{N}=$ neutral; $\mathrm{NA}=$ not available.

${ }^{b} \mathrm{MAF}$ (minor allele frequency) values derive from gnomAD and are considered rare if $\mathrm{MAF}<0.001$ when occurring in heterozygosis.

'Semen analysis was performed for all patients (Azoo = azoospermia; Crypto = cryptozoospermia), and if biopsies were available, patients' phenotypes were also histologically assessed (SCO = Sertoli cell-only syndrome; MeiA = meiotic arrest; Hypo = hypospermatogenesis).

${ }^{\mathrm{d}}$ Testicular volumes (testis vol., right/left, ref. $>15 \mathrm{~mL}$ each; bold = values outside the normal range).

eLuteinizing hormone ( $\mathrm{LH}$, ref. 2-10 IU/L; bold = values outside the normal range).

${ }^{f}$ Follicle-stimulating hormone (FSH, ref. 1-7 IU/L; bold = values outside the normal range).

'Testosterone ( $\mathrm{T}$, ref. $>12 \mathrm{nmol} / \mathrm{L}$; bold = values outside the normal range). 


\begin{tabular}{|c|c|c|c|c|c|c|c|c|c|c|}
\hline Variant (cDNA) & Variant (protein) & $\begin{array}{l}\text { Predicted } \\
\text { effect }^{\mathrm{a}}\end{array}$ & MAF $^{b}$ & $\begin{array}{l}\text { Subject } \\
\text { ID }\end{array}$ & Age \& origin & $\begin{array}{l}\text { Semen analysis/ } \\
\text { histologyc }\end{array}$ & $\begin{array}{l}\text { Testis } \\
\text { vol. }^{d}\end{array}$ & $\mathbf{L H}^{\mathbf{e}}$ & $\mathrm{FSH}^{\mathrm{f}}$ & $T^{g}$ \\
\hline c.224_240dup & p.(Gly81CysfsTer24) & NA & 0 & M364 & 26; Germany & Azoo/SCO & $24 / 21$ & 9 & 17.9 & 7.6 \\
\hline c. $248 \mathrm{C}>\mathrm{T}$ & p.(Ala83Val) & $10.2 ; \mathrm{B} / \mathrm{T} / \mathrm{N}$ & 0 & M2118 & 33; Germany & Crypto/NA & $16 / 17$ & 4.1 & 7.6 & 14.1 \\
\hline c. $368 \mathrm{~T}>\mathrm{C}$ & p.(Val123Ala) & 18.5; D/D/D & 0 & M2110 & 43; Kazakstan & Crypto/NA & $19 / 17$ & 5.5 & 6.2 & 14.9 \\
\hline c. $553 \mathrm{G}>\mathrm{T}$ & p.(Ala185Ser) & $0.01 ; \mathrm{B} / \mathrm{T} / \mathrm{N}$ & 0.0047 & M1325 & 33; Germany & Azoo/Hypo & $7 / 8$ & 4.6 & 6.8 & 15.6 \\
\hline c. $553 \mathrm{G}>\mathrm{T}$ & p.(Ala185Ser) & $0.01 ; \mathrm{B} / \mathrm{T} / \mathrm{N}$ & 0.0047 & M1562 & 36; Germany & Azoo/SCO & $9 / 6$ & 7.3 & 24.7 & 13.8 \\
\hline c. $785 \mathrm{G}>\mathrm{C}$ & p.(Gly262Ala) & $0.2 ; \mathrm{B} / \mathrm{T} / \mathrm{D}$ & 0 & M2173 & 28, Turkey & Azoo/SCO & $13 / 18$ & 2.9 & 8.3 & 17.7 \\
\hline c. $803 \mathrm{~T}>\mathrm{A}$ & p.(Leu268His) & 15.6; D/T/D & 0 & M1793 & 33; Turkey & Azoo/MeiA & $15 / 15$ & 6.3 & 8.4 & 15.3 \\
\hline c. $1070 \mathrm{C}>\mathrm{T}$ & p.(Ala357Val) & 23.2; B/T/D & 0.0001 & M1686 & 29; Romania & Crypto/NA & $17 / 16$ & 9.5 & 7.1 & 39.7 \\
\hline c. $1486 C>T$ & p.(Arg496Cys) & 28.8; D/D/D & 0.0001 & M1083 & 36; Germany & Azoo/NA & $12 / 8$ & 9.6 & 18.1 & 17.5 \\
\hline c. $1549 \mathrm{C}>\mathrm{T}$ & p.(Arg517Cys) & $23.4 ; \mathrm{D} / \mathrm{T} / \mathrm{D}$ & 0.0016 & M468 & 34; Pakistan & Azoo/MeiA & $19 / 16$ & 6.8 & 6.4 & 19.8 \\
\hline c. $1613 C>G$ & p.(Ala538Gly) & $14.4 ; \mathrm{B} / \mathrm{T} / \mathrm{D}$ & 0 & M2069 & 29; Lebanon & Azoo/NA & $9 / 6$ & 3.5 & 8.4 & 6.1 \\
\hline c. $1789 \mathrm{G}>\mathrm{A}$ & p.(Gly597Ser) & 30; D/D/D & 0.0022 & M244 & 31; Germany & Azoo/MeiA & $26 / 26$ & 2.8 & 5.2 & 15.2 \\
\hline c. $1789 \mathrm{G}>\mathrm{A}$ & p.(Gly597Ser) & 30; D/D/D & 0.0022 & M754 & 32; Turkey & Azoo/SCO & $6 / 7$ & 11 & 30.7 & 15.9 \\
\hline c. $1789 \mathrm{G}>\mathrm{A}$ & p.(Gly597Ser) & $30 ; \mathrm{D} / \mathrm{D} / \mathrm{D}$ & 0.0022 & M2141 & 27; Turkey & Azoo/Hypo & $17 / 14$ & 5.8 & 13.5 & 16.6 \\
\hline
\end{tabular}


Figure 1

A

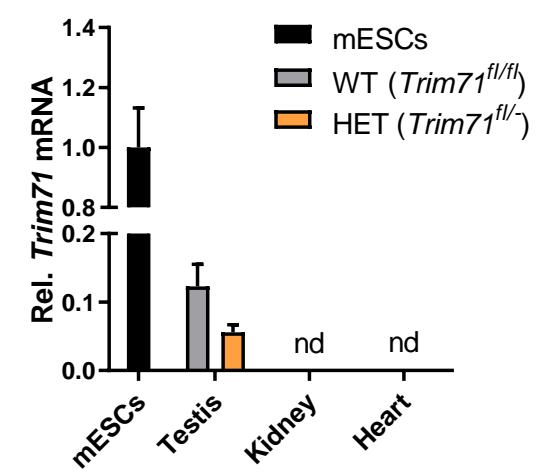

D
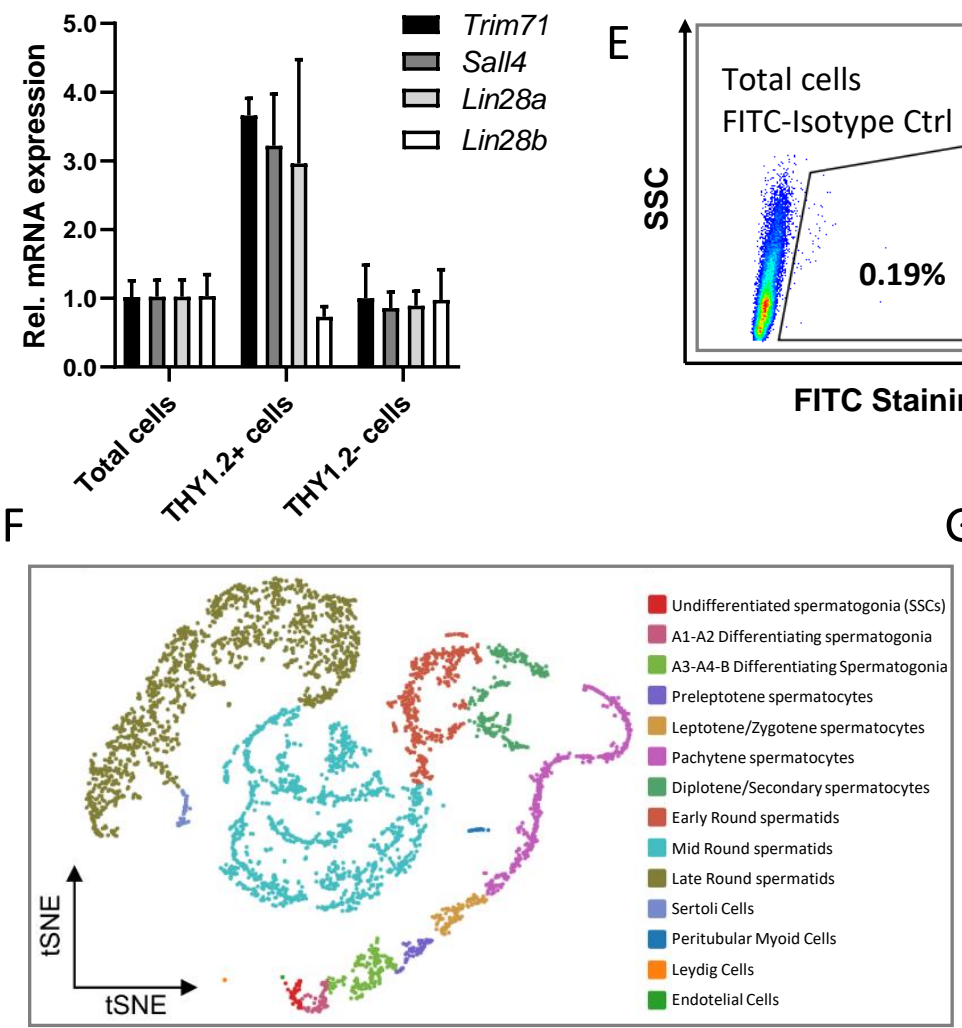

B

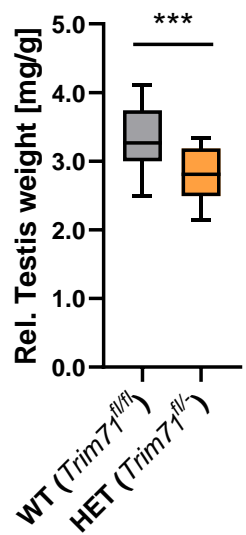

$\mathrm{E}$

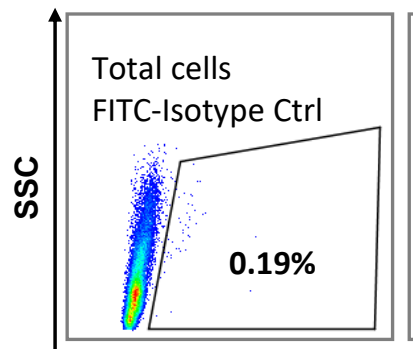

FITC Staining

C
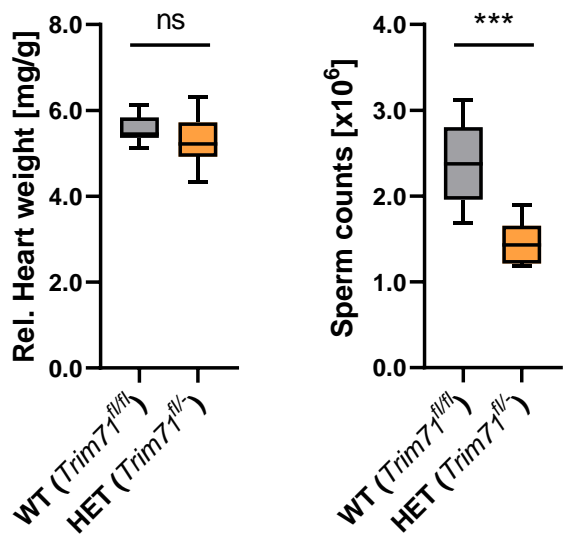

Total cells
FITC-THY1.2/CD90.2

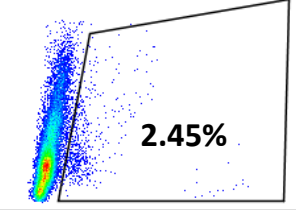

MACS populations

FITC-selected

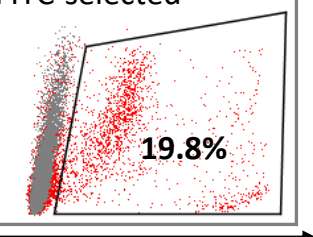

FITC-Negative selection (THY1.2- cells)

FITC-Positive selection (THY1.2+ cells)

G

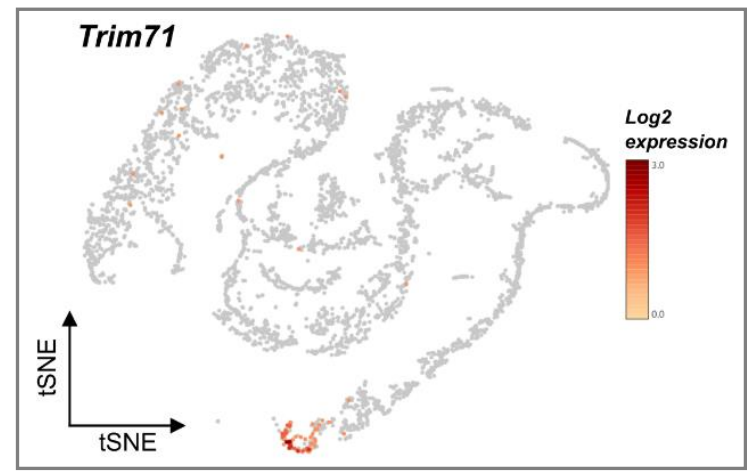

$\mathrm{H}$
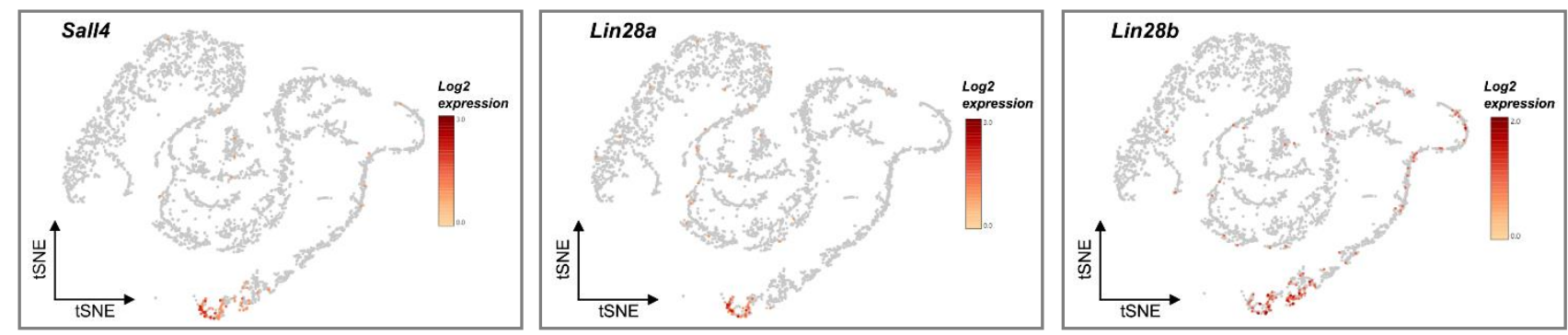
Figure 1. Trim71 is expressed in spermatogonial stem cells (SSCs) of adult fertile mice. A) qRT-PCR of Trim 71 relative to Hprt housekeeping gene in testis, kidney and heart of wild type (WT, Trim71 fl/fl) and Trim 71 heterozygous (HET, Trim 71 ${ }^{\mathrm{fl} / \mathrm{-}}$ ) adult male mice (10-14 weeks old), normalized to Trim71 relative levels in wild type murine ESCs ( $n d=$ not detected). Error bars represent SD $(n=3)$. B) Organ weight [mg] relative to total body weight $[\mathrm{g}]$ of testis, kidney and heart of wild type (WT, Trim $71^{f / f l}$ ) and Trim71 heterozygous (HET, Trim71 fl/-) male adult mice (10-14 weeks old). Graphs represent Tukey plots ( $\mathrm{n}=14-$ 16). ${ }^{* * *}$ P-value $<0.005$, ns = non-significant (unpaired Student's t-test). C) Epididymal sperm counts in wild type (WT, Trim71 ${ }^{f / f l}$ ) and Trim71 heterozygous (HET, Trim71 fl/-) male adult mice (10-14 weeks old). ***P-value < 0.005 (unpaired Student's t-test). D) qRT-PCR of Trim71, the SSC markers Sall4 and Lin28a, and the differentiating spermatogonia marker Lin28b, relative to Hprt housekeeping gene in testes cell suspension before (total cells) and after THY1.2 MACS (THY1.2+/- cells). Error bars represent SD (n=3). E) Representative flow cytometry scatter plots of testes cell suspension before (total cells) and after THY1.2/CD90.2 MACS (Thy1.2+/- cells). F) tSNE plot (t-distributed stochastic neighbor embedding) of single-cell transcriptome data (scRNA-seq) from mouse testes as published by Hermann et al., 2018. Each dot represents a single cell and is colored according to its cluster identity as indicated on the figure key. G) Expression pattern of Trim71 and H) Sall4, Lin28a and Lin28b projected on the tSNE plot of the mouse scRNA-seq dataset. Red indicates high expression and gray indicates low or no expression, as shown by the figure key. See also Suppl. Fig. 1. 


\section{Figure 2}

A

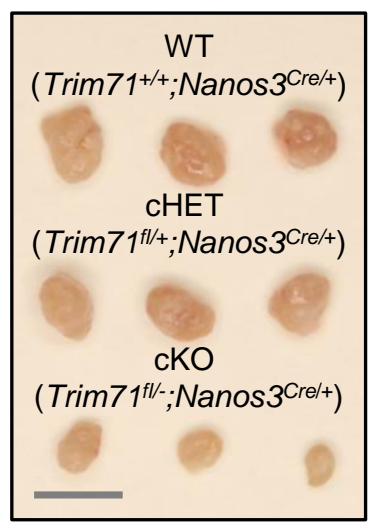

B

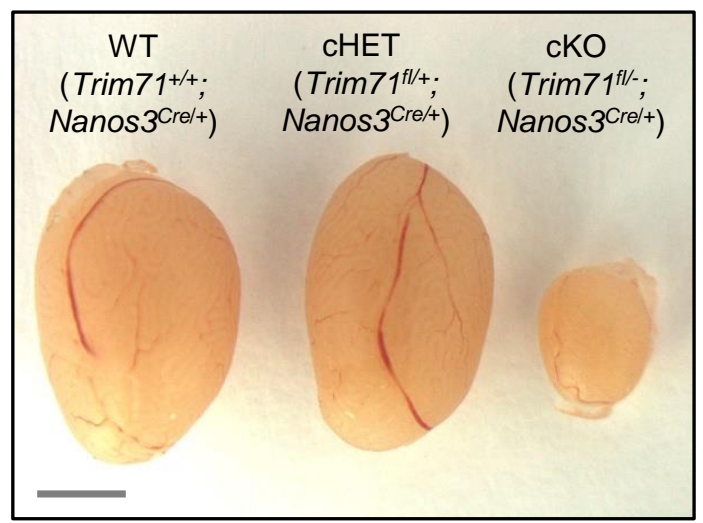

C

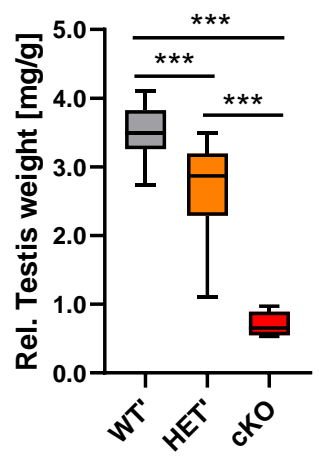

D
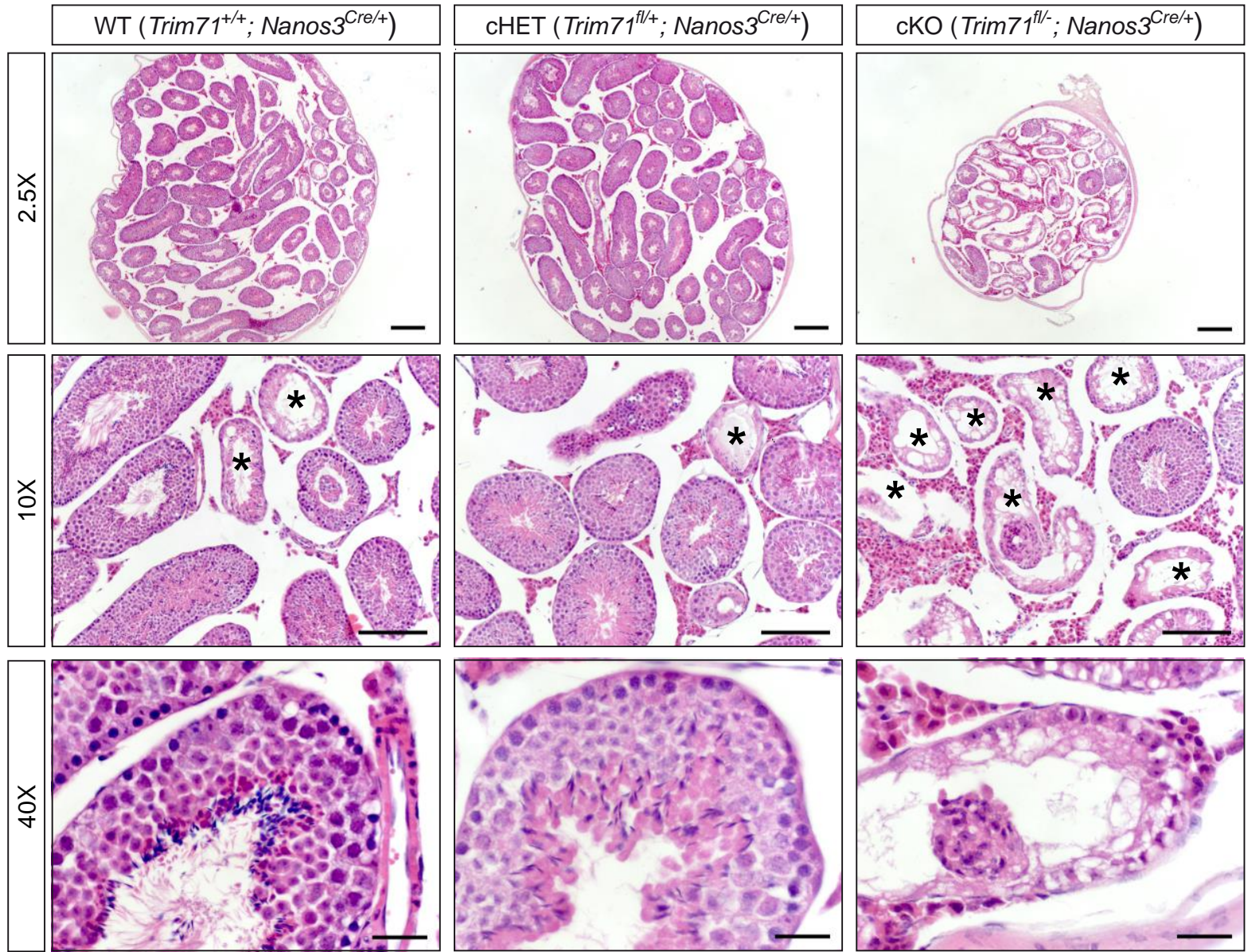
E
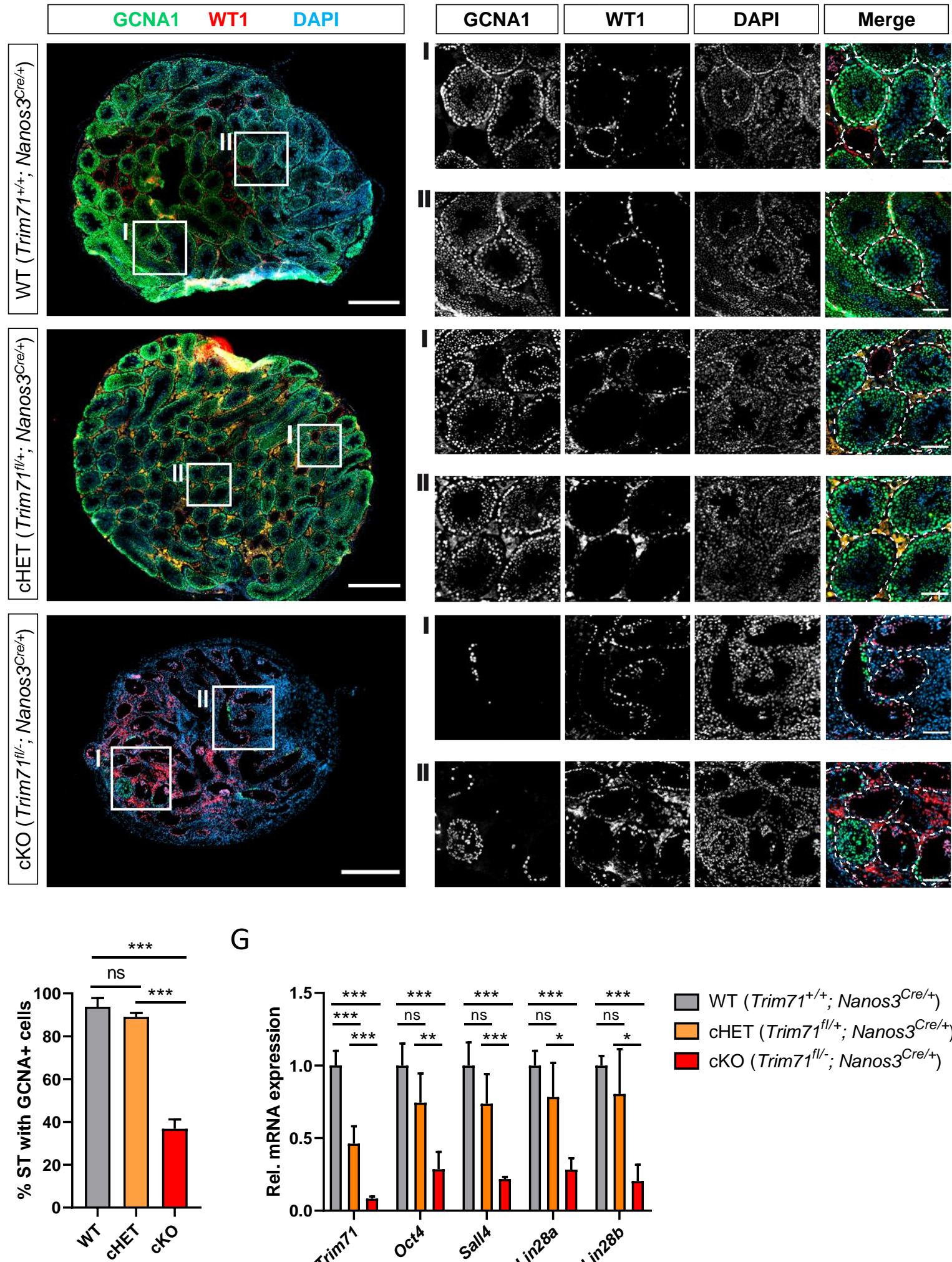

G

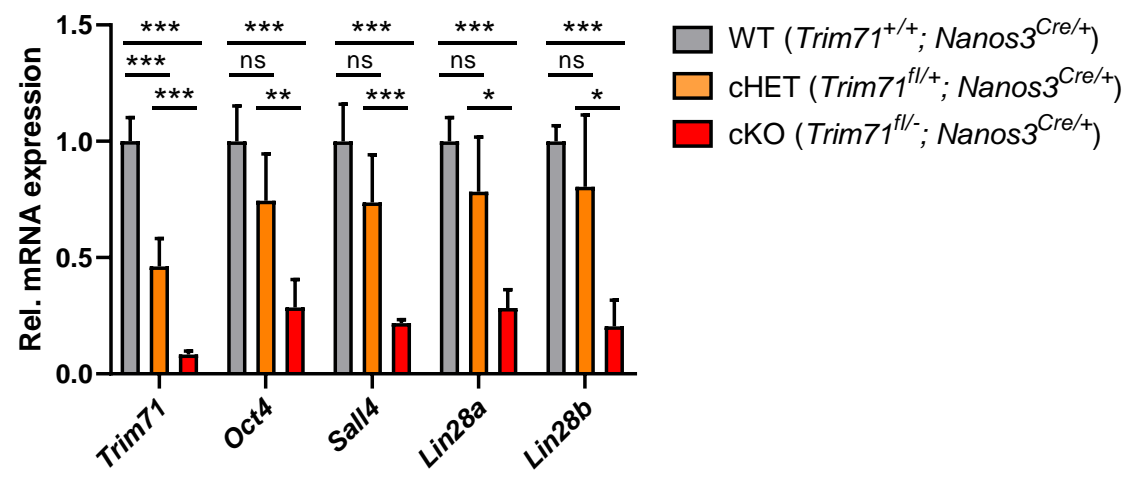


Figure 2. Germline-specific Trim71 cKO male mice present a Sertoli cell-only (SCO) phenotype. A) Representative images of ovaries and B) testes of adult wild type (WT, Trim $71^{+/+}$; Nanos $3^{\mathrm{Cre} /+}$ ), germlinespecific Trim 71 heterozygous (cHET, Trim $71^{\mathrm{fl} /+}$; Nanos $3^{\mathrm{Cre} /+}$ ) and germline-specific Trim 71 knockout (cKO, Trim $71^{\mathrm{fl} /-}$; Nanos3 ${ }^{\mathrm{Cre} /+}$ ) mice. Scale bars represent $2 \mathrm{~mm}$. C) Testis weight [mg] relative to total body weight [g] of wild type (WT'), Trim71 heterozygous (HET') and germline-specific Trim71 knockout (cKO) mice, summarized from Suppl. Fig. 2C, joining several Nanos3 genotypes per Trim71 genotype. Error bars represent SD ( $\mathrm{n}=8-29)$. *** P-value < 0.005 (one-way ANOVA, Tukey's test). D) Representative H\&E stainings on paraffin testes cross-sections from adult wild type (WT, Trim $71^{+/+}$; Nanos $3^{\mathrm{Cre} /+}$ ), germlinespecific Trim 71 heterozygous (cHET, Trim $71^{\mathrm{fl} /+}$; Nanos3 ${ }^{\mathrm{Cre} /+}$ ) and germline-specific Trim 71 knockout (cKO, Trim $71^{\mathrm{fl} /-}$; Nanos $3^{\mathrm{Cre} /+}$ ) mice. Seminiferous tubules with a defective morphology are marked with an asterisk $\left(^{*}\right)$ in 10x magnification images. Scale bars represent $200 \mu \mathrm{m}, 100 \mu \mathrm{m}$ and $20 \mu \mathrm{m}$ in $2.5 \mathrm{x}, 10 \mathrm{x}$ and 40x magnifications, respectively. E) Representative immunofluorescence stainings on testes cryosections from adult wild type (WT, Trim $71^{+/+} ;$Nanos $^{\mathrm{Cre} /+}$ ), germline-specific Trim71 heterozygous (cHET, Trim71 $1^{\mathrm{fl} /+}$; Nanos3 ${ }^{\mathrm{Cre} /+}$ ) and germline-specific Trim 71 knockout (cKO, Trim71 $1^{\mathrm{fl} /-;}$; Nanos3 ${ }^{\mathrm{Cre} /+}$ ) mice. Images show co-staining with GCNA1 (germ cells), WT1 (Sertoli cells) and DAPI (nuclei). For each genotype two regions - indicated as I and II - are depicted in higher magnification. Scale bars represent $500 \mu \mathrm{m}$ for complete testes cross-sections and $100 \mu \mathrm{m}$ for the magnification images. F) Quantification of seminiferous tubules (ST) containing GCNA+ cells per testis cross-section from E, depicted as percentages. Error bars represent SD $(n=3)$. ***P-value $<0.005, \mathrm{~ns}=$ non-significant (unpaired Student's t-test). G) qRT-PCR of Trim71, the SSC markers Sall4, Lin28a and Oct4, and the differentiating spermatogonia marker Lin28b, relative to Hprt housekeeping gene in whole testis RNA of wild type (WT, Trim71+/+; Nanos $3^{\mathrm{Cre} /+}$ ), germline-specific Trim71 heterozygous (cHET, Trim $71^{\mathrm{fl} /+}$; Nanos $3^{\mathrm{Cre} /+}$ ) and germline-specific Trim 71 knockout (cKO, Trim $71^{\mathrm{fl} /-}$; Nanos $3^{\mathrm{Cre} /+}$ ) male adult mice. Error bars represent SD (n=3-6). ***P-value < 0.005; **P-value < 0.001; *P-value < 0.05; ns = non-significant (unpaired Student's t-test). See also Suppl. Fig. 2. 


\section{Figure 3}

A
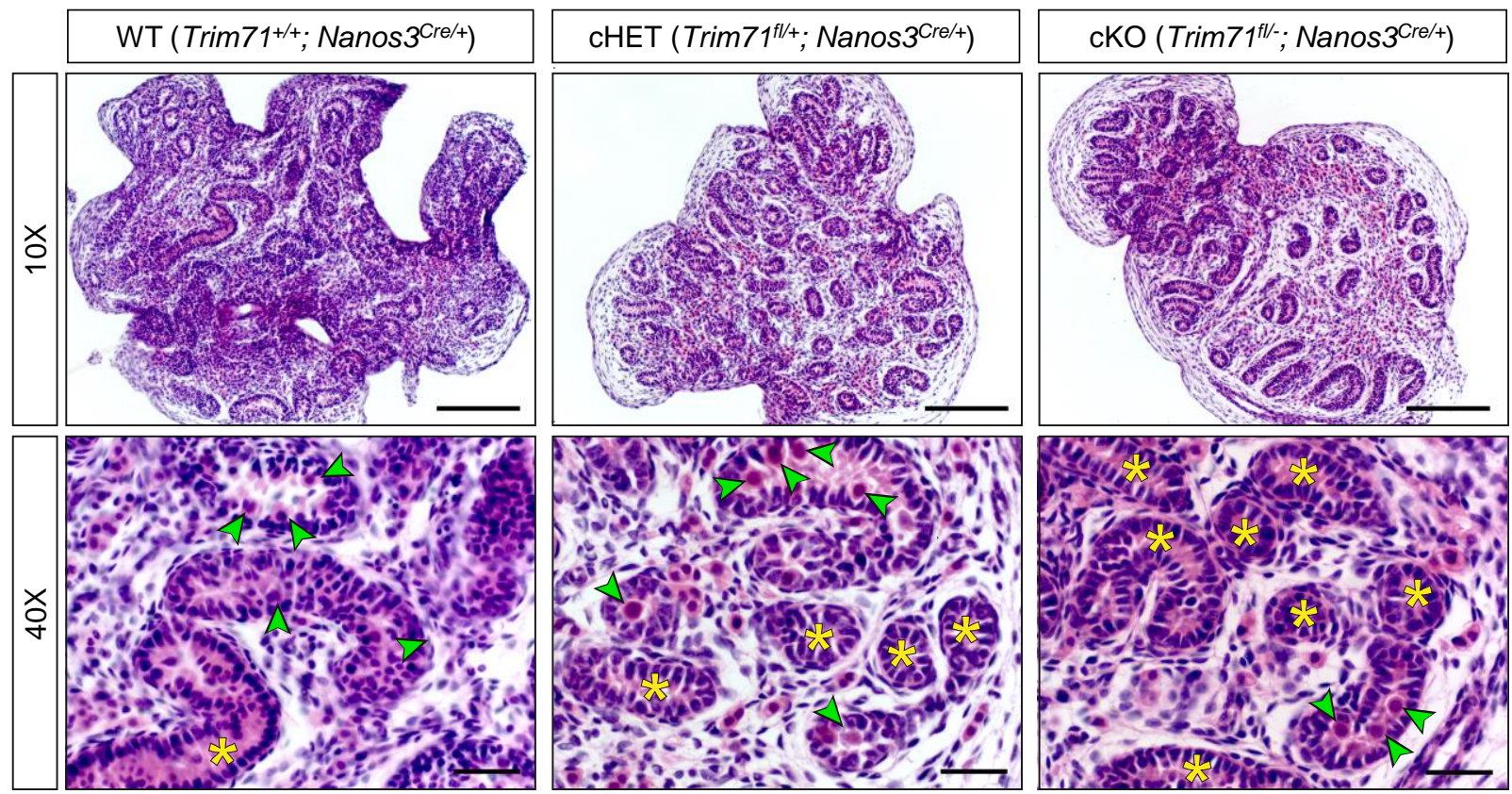

B
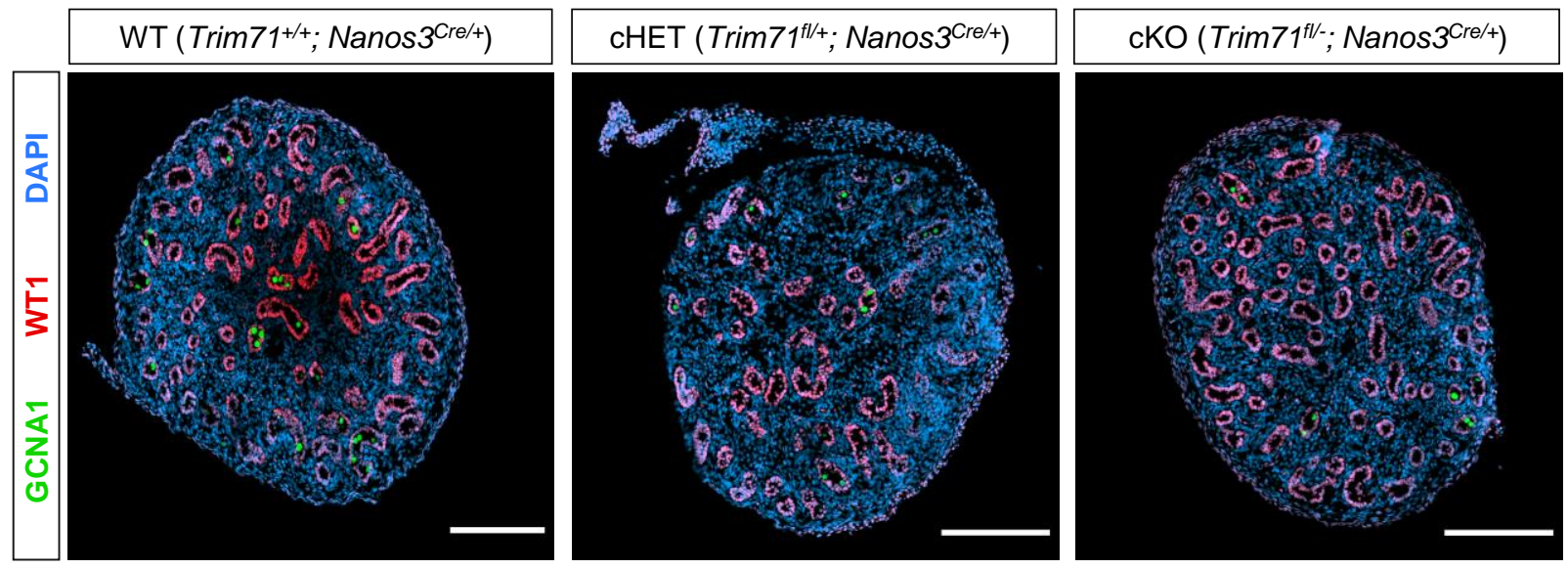

C

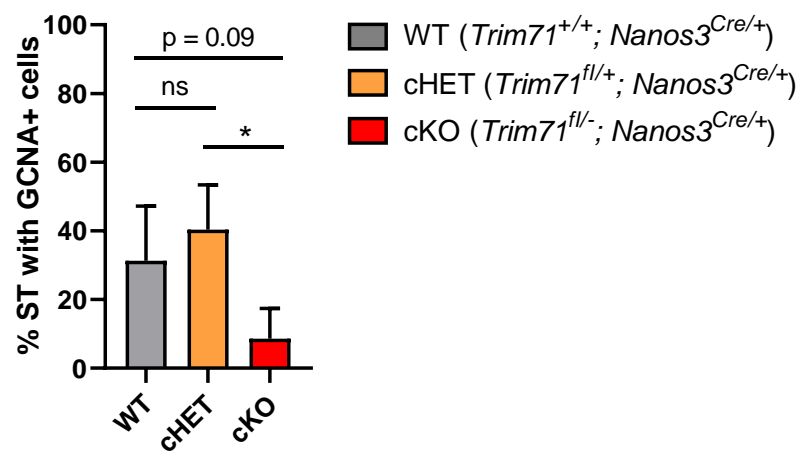


Figure 3. Infertility in germline-specific Trim71 cKO male mice has an embryonic origin. A) Representative H\&E stainings on paraffin testes cross-sections from neonatal (P0.5) wild type (WT, Trim $71^{+++}$; Nanos $3^{\mathrm{Cre} /+}$ ), germline-specific Trim 71 heterozygous (CHET, Trim $71^{\mathrm{fl} /+} ;$ Nanos $3^{\mathrm{Cre} /+}$ ) and germline-specific Trim 71 knockout (cKO, Trim $71^{\mathrm{fl} /-}$; Nanos3 ${ }^{\mathrm{Cre} /+}$ ) male mice. Gonocytes within the seminiferous tubules are marked with a green arrow head, and gonocyte-lacking seminiferous tubules are marked with a yellow asterisk $\left({ }^{*}\right)$ in $40 x$ magnification images. Scale bars represent $100 \mu \mathrm{m}$ and 20 $\mu \mathrm{m}$ in in 10x and 40x magnifications, respectively. B) Representative immunofluorescence stainings on testes cryo-sections from neonatal (PO.5) wild type (WT, Trim $71^{+/+}$; Nanos $3^{\mathrm{Cre} /+}$ ), germline-specific Trim 71 heterozygous (cHET, Trim $71^{\mathrm{fl} /+} ;$ Nanos $3^{\mathrm{Cre} /+}$ ) and germline-specific Trim 71 knockout (cKO, Trim $71^{\mathrm{fl} /-}$; Nanos $3^{\mathrm{Cre} /+}$ ) male mice. Images show co-staining with GCNA1 (gonocytes), WT1 (Sertoli cells) and DAPI (nuclei). Scale bars represent $200 \mu \mathrm{m}$. C) Quantification of seminiferous tubules (ST) containing GCNA+ cells per testis cross-section from B, depicted as percentages. Error bars represent SD (n=3). *Pvalue $<0.05, \mathrm{~ns}=$ non-significant (unpaired Student's t-test). See also Suppl. Fig. 2. 
Figure 4

A
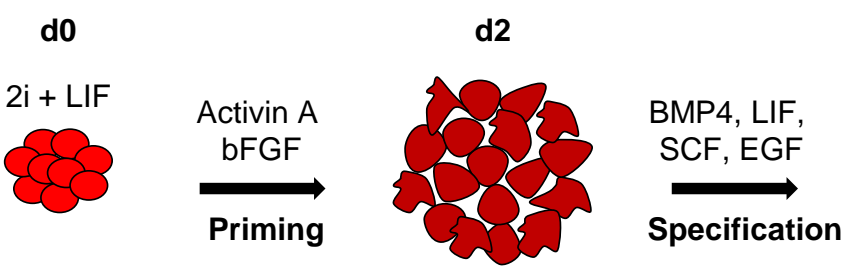

d8
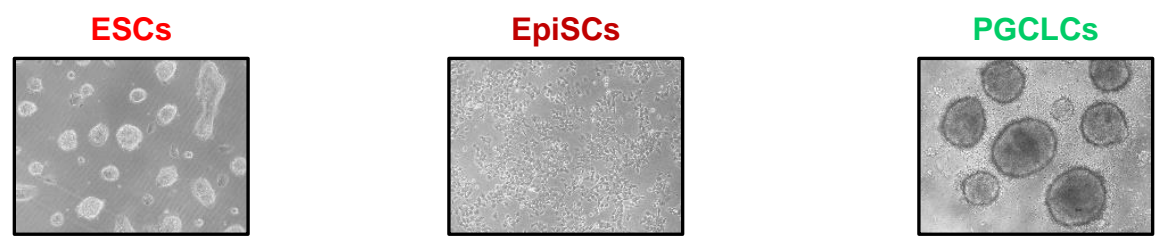

B

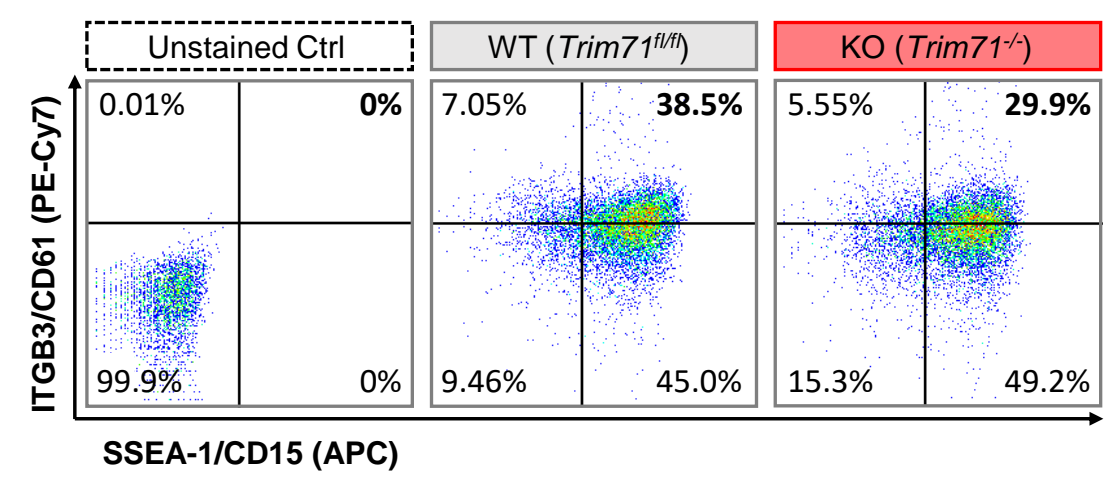

C

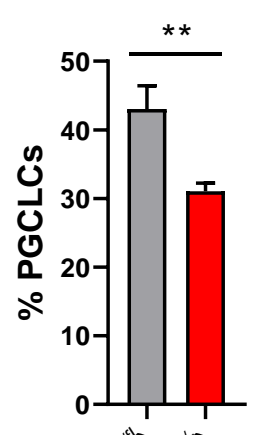

D

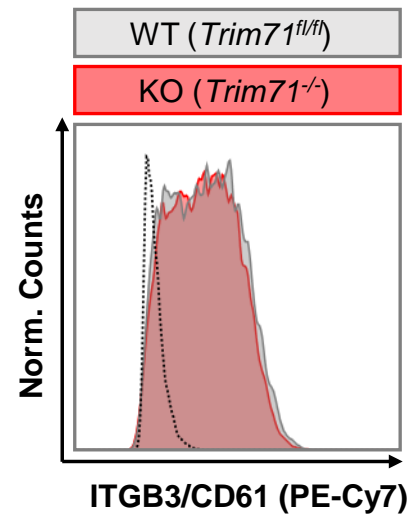

E

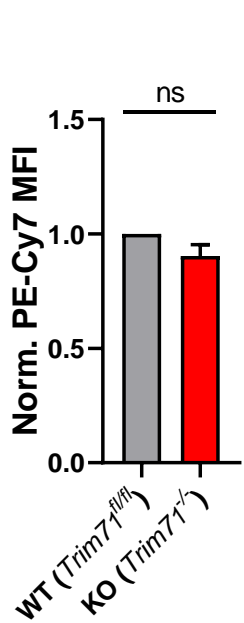

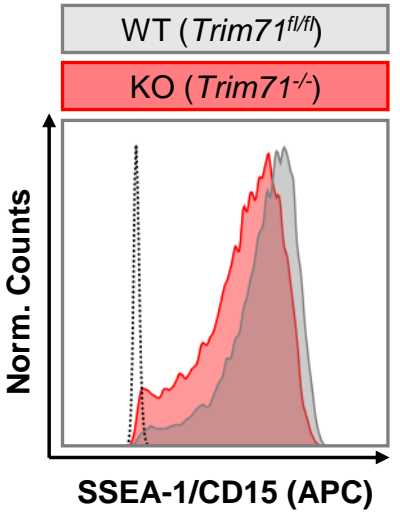
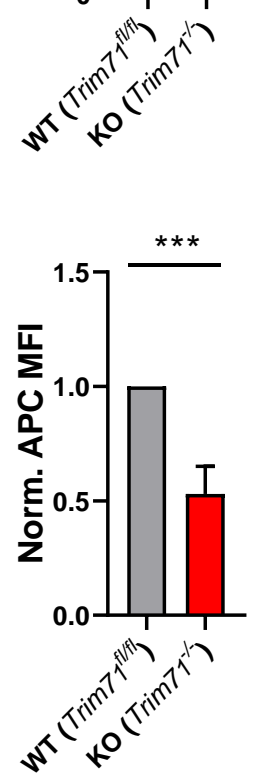
Figure 4. Trim71-deficient mouse ESCs show an impairment during PGC specification. A) Schematic representation of the methodology for the in vitro differentiation of murine ESCs into PGC-like cells (PGCLCS) accompanied by representative microscopic images (10x magnifications) of cells at different stages of the differentiation process (d0, d2 and d8). B) Representative flow cytometry scatter plots from d8 cells derived from wild type (WT, Trim71 fl/fl) and Trim71 knockout (KO, Trim $71^{-/}$) mouse ESCs stained for the PGC-specific surface markers ITGB3/CD61 (PE-Cy7) and SSEA-1/CD15 (APC). Double positively stained cells are considered PGCLCs. C) Quantification of PGCLCs in several experimental replicates of B. Error bars represent SEM (n=6). D) Representative histograms for individual ITGB3/CD61 (PE-Cy7) and E) SSEA-1/CD15 (APC) staining of d8 cells derived from wild type (WT, Trim71 $1^{f / f l}$ ) and Trim 71 knockout (KO, Trim $71 \%$ ) murine ESCs accompanied by the median fluorescence intensity (MFI) quantification for the respective staining in replicate experiments. Error bars represent SEM ( $n=6)$. ***Pvalue $<0.005 ; * *$ P-value $<0.001 ; n s=$ non-significant (unpaired Student's t-test). See also Suppl. Fig. 3. 


\section{Figure 5}

A

NCCIT EV:ARING (1:1)

Wild type reads

Reads with frameshift mutations

$\square$ Reads with in-frame mutations

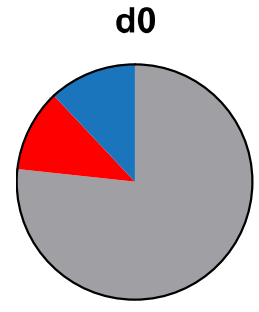

$76.69 \%$

$11.34 \%$

$11.97 \%$

Total $=12720$

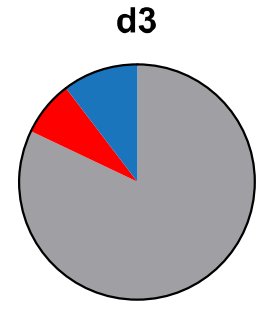

$82.10 \%$

$7.53 \%$

$10.37 \%$

Total $=13941$

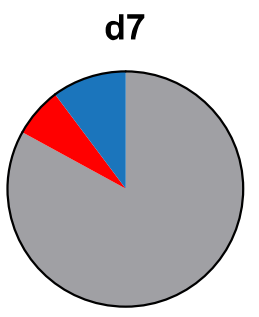

$82.97 \%$

$6.82 \%$

$10.21 \%$

Total $=14170$

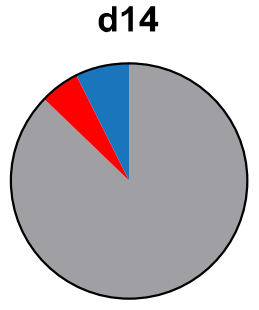

$87.23 \%$

$5.37 \%$

$7.40 \%$

Total $=12593$

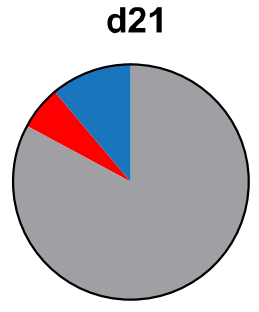

$82.91 \%$

$5.96 \%$

$11.13 \%$

Total $=19608$

B

\section{NCCIT EV:ANHL6 (1:1)}

\section{Wild type reads}

$\square$ Reads with frameshift mutations

$\square$ Reads with in-frame mutations

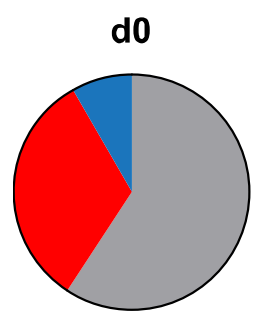

$59.26 \%$

$32.46 \%$

$8.28 \%$

Total $=30475$

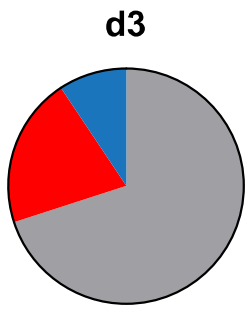

$70.05 \%$

$20.60 \%$

$9.35 \%$

Total $=39632$

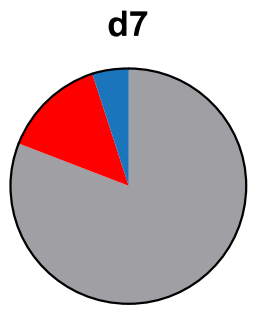

$80.92 \%$

$14.11 \%$

$4.97 \%$

Total $=22664$

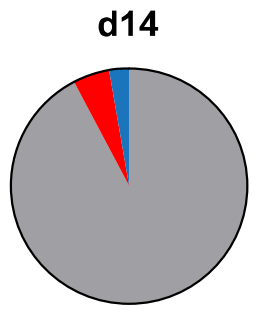

$92.30 \%$

$4.95 \%$

$2.75 \%$

Total $=48009$

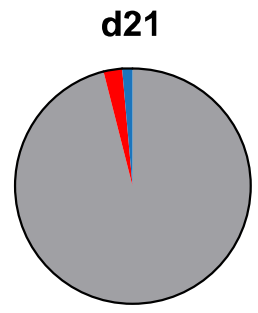

$96.06 \%$ $2.53 \%$

$1.41 \%$

Total $=31663$ 
Figure 5. NCCIT cells with TRIM71 mutations show proliferation defects in growth competition assays. A) Pie charts showing allele frequencies for wild type (EV) and TRIM71 RING mutant ( $\triangle R I N G)$ or B) TRIM71 NHL mutant ( $\triangle \mathrm{NHL6}$ ) NCCIT cells obtained at different time points during growth competition assays. For the assay, NCCIT wild type cells (EV) were mixed 1:1 with either TRIM71 RING mutant $(\triangle R I N G)$ or TRIM71 NHL mutant ( $\triangle \mathrm{NHL6})$ NCCIT cells and directly analyzed (d0) before their culturing, following by subsequent analysis at several time points (d3, d7, d14 and d21). Allele frequency was analyzed via NGS using the Illumina MiSeq platform. For TRIM71 mutant alleles, reads with in-frame mutations and frameshift mutations (loss-of-function) in each respective domain (RING or NHL) are depicted. The total number of sequencing reads for each time point is indicated under each respective pie chart. See also Suppl. Fig. 4 and 5. 


\section{Figure 6}

A

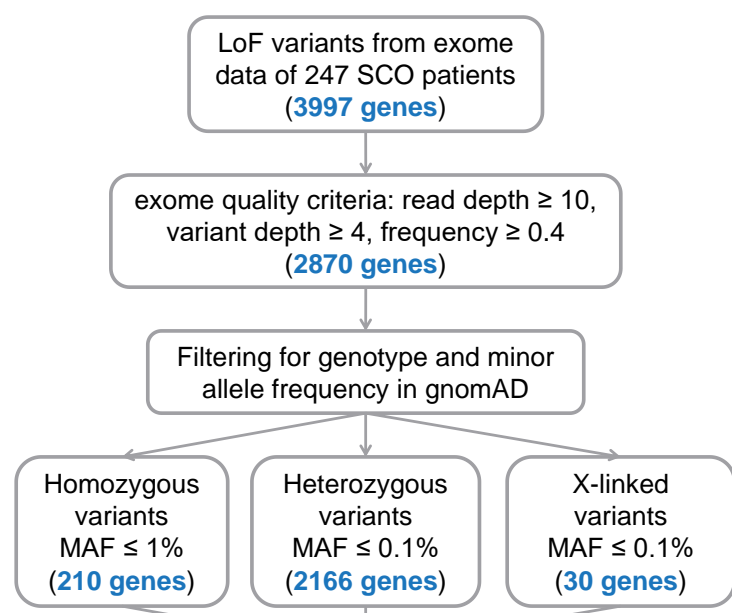

High expression in testis (GTEx)

(949 genes)

Exclusion of genes with LoF variants in control subjects with intact spermatogenesis (721 genes including TRIM71)
B

Genes with LoF variants in $\mathrm{X}$ patients $\square=2 \square 3 \square 4$

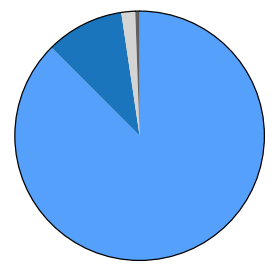

$87.52 \%$ $10.13 \%$ $1.80 \%$

$0.55 \%$

Total $=721$

C Genes with LoF variants in $\mathrm{X}$ patients $\square=2 \square 3 \square 4 \square 5 \square 6$

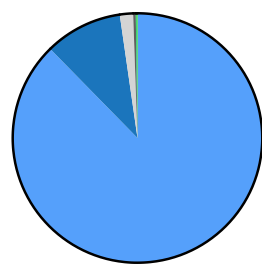

$87.67 \%$ $10.06 \%$

$1.72 \%$

$0.33 \%$

$0.11 \%$

$0.11 \%$

Total $=1800$

D

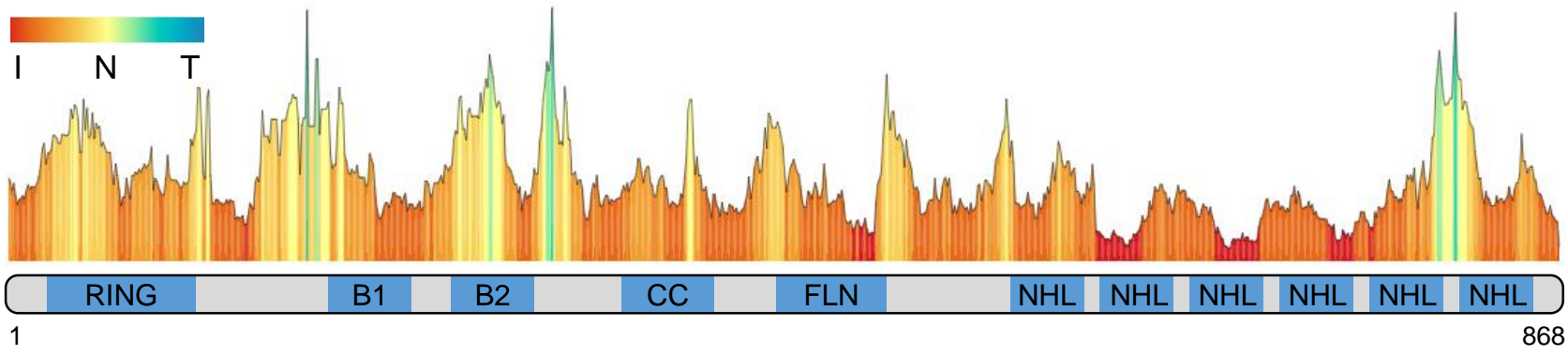

$\begin{array}{llllll}E & \text { A83V V123A } & \text { G262A } & \text { A357V } & \text { R517C } & \text { G597S }\end{array}$

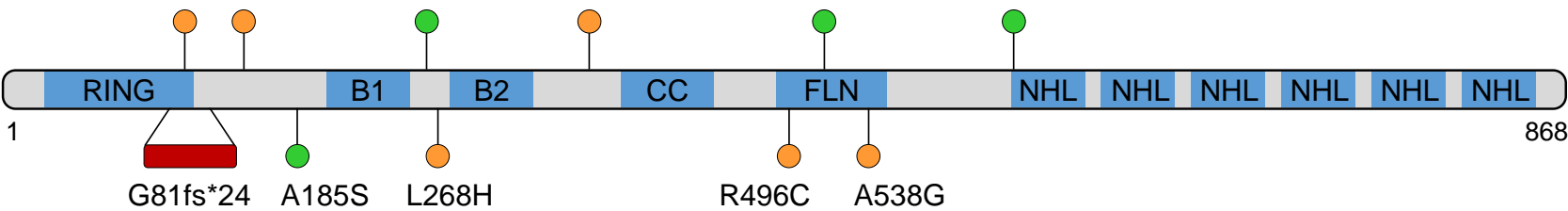

$\mathrm{F}$

Control Patient

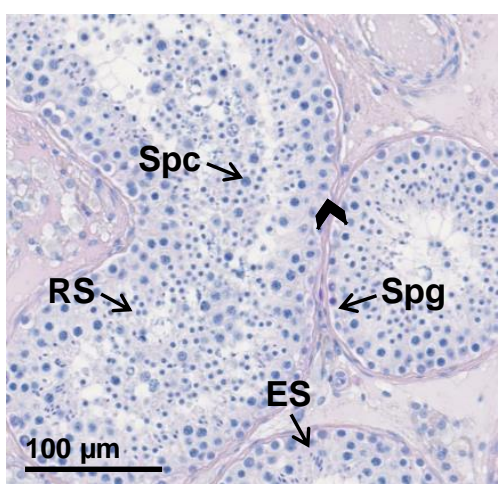

Patient M364 - TRIM71 LoF c224_240dup/p.(Gly81CysfsTer24)

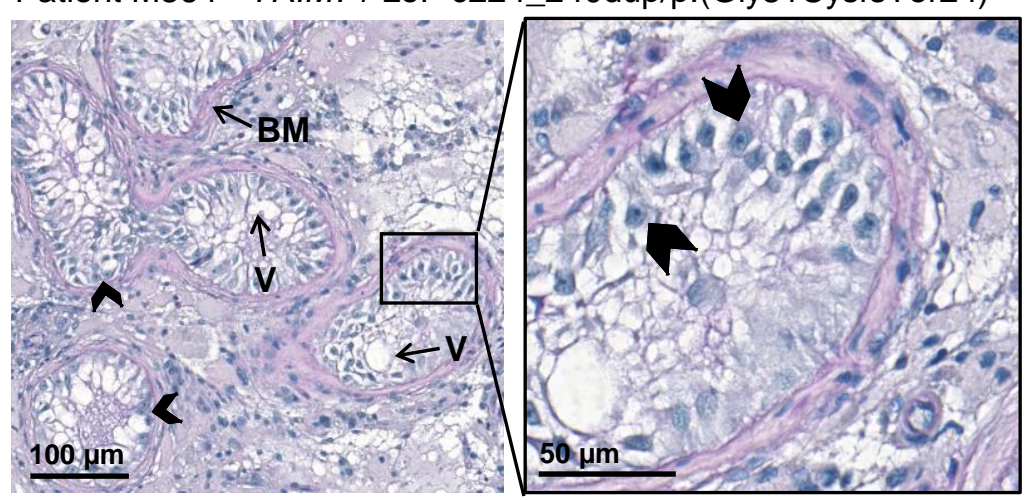


Figure 6. Exome sequencing in infertile SCO patients reveals an association of TRIM71 with human male infertility. A) Filtering scheme detailing gene prioritization of exome sequencing data from 247 individuals with Sertoli cell-only (SCO) phenotype using Sciobase and Haystack (see details in the Methods section). Gene numbers remaining after each filtering step are indicated in blue. Loss-offunction (LoF) variants include stop, frameshift, splice acceptor and splice donor variants. gnomAD = Genome Aggregation Database; MAF = minor allele frequency; GTEx = Genotype-Tissue Expression Portal. B) Percentages of genes (total $=721$, obtained at the end of the filtering process depicted in A) affected by LoF variants in 1-4 SCO patients. None of the genes carried LoF variants in more than 4 patients in the SCO cohort $(n=247)$. C) Percentages of genes (total $=1800$, obtained at the end of the filtering process depicted in A, excluding "High expression in testis - GTEx" filtering step) affected by LoF variants in 1-6 SCO patients. None of the genes carried LoF variants in more than 6 patients in the SCO cohort ( $\mathrm{n}=247)$. D) TRIM71 mutation tolerance landscape obtained from MetaDome (https://stuart.radboudumc.nl/metadome/). The graph displays missense over synonymous variant ratios per position for the entire protein, based on gnomAD variants. $\mathrm{I}=$ intolerant; $\mathrm{N}=$ neutral; $\mathrm{T}=$ tolerant. E) Schematic representation of TRIM71 domain structural organization depicting the location of the genetic variants identified in MERGE. Red = presumably pathogenic LoF variants; orange = missense variants of uncertain significance; green = missense variants found also in proven fathers. F) Periodic acid-Schiff stainings of testis sections obtained by testicular biopsy from a control patient and an SCO patient (subject M364), who harbors the TRIM71 variant c.224_240dup/p.(Gly81CysfsTer24) in heterozygosis. The control section shows intact spermatogenesis (Spg = spermatogonia; $\mathrm{Spc}=$ spermatocytes; RS = round spermatids; $\mathrm{ES}$ = elongated spermatids) as opposed to subject M364, whose histological evaluation revealed an SCO phenotype (exemplary Sertoli cells indicated by arrow heads) with several degenerated seminiferous tubules, frequently including large vacuoles (V) and thickened basement membranes (BM). See also Suppl. Fig. 6. 